\title{
Structural insights into the small G-protein Arl13B and implications for Joubert syndrome
}

\author{
Mandy MIERTZSCHKE* ${ }^{\star}$, Carolin KOERNER ${ }^{\star}$, Michael SPOERNER $\dagger$ and Alfred WITTINGHOFER ${ }^{\star 1}$ \\ *Emeritus group A. Wittinghofer, Max-Planck-Institute for Molecular Physiology, BMZ, Otto-Hahn-Straße 15, 44227 Dortmund, Germany \\ $\dagger$ †nstitute of Biophysics and Physical Biochemistry, University of Regensburg, Universitätsstraße 31, 93053 Regensburg, Germany
}

\begin{abstract}
Ciliopathies are human diseases arising from defects in primary or motile cilia. The small G-protein Arl13B (ADP-ribosylation factor-like 13B) localizes to microtubule doublets of the ciliary axoneme and is mutated in Joubert syndrome. Its GDP/GTP mechanistic cycle and the effect of its mutations in patients with Joubert syndrome remain elusive. In the present study we applied high resolution structural and biochemical approaches to study Arl13B. The crystal structure of Chlamydomonas rheinhardtii Arl13B, comprising the G-domain and part of its unique C-terminus, revealed an incomplete active site, and together with biochemical data the present study accounts for the absence of intrinsic GTP hydrolysis by this protein. The structure
\end{abstract}

shows that the residues representing patient mutations $\mathrm{R} 79 \mathrm{Q}$ and R200C are involved in stabilizing important intramolecular interactions. Our studies suggest that $\mathrm{Arg}^{79}$ is crucial for the GDP/GTP conformational change by stabilizing the large tworesidue register shift typical for Arf (ADP-ribosylation factor) and Arl subfamily proteins. A corresponding mutation in Arl3 induces considerable defects in effector and GAP (GTPase-activating protein) binding, suggesting a loss of Arl13B function in patients with Joubert syndrome.

Key words: Arl13B, Arl/Arf G-protein, cilia, GTPase activity, interswitch toggle, Joubert syndrome patient mutation.

\section{INTRODUCTION}

Cilia are small microtubule-based organelles emanating from almost every cell type of the human body [1]. Cilia fulfil crucial sensory functions and are a central compartment in a range of signalling pathways, including Sonic Hedgehog and Wnt signalling [2,3]. Ciliopathies are diseases originating from the disruption of the structural integrity of cilia and/or their signalling pathways [4,5]. Arl13B (ADP-ribosylation factor-like 13B) is one of several proteins mutated in Joubert syndrome [6], a ciliopathy characterized by brain malformations with its characteristic molar tooth sign, combined with polydactyly and formation of kidney cysts [7]. Arl13B is considered a ciliary marker, as it exclusively localizes to the microtubule doublets of the ciliary axoneme. Gene knockouts in mouse [8], Caenorhabditis elegans [9] and zebrafish $[10,11]$ confirmed its importance for cilia biology. Loss of Arl13B in mice leads to failure of B-tubule closure in the ciliary axoneme [8] and in C. elegans to mislocalization of various sensory receptors such as PKD2 (protein kinase D2), ODR-10, Tax2 and OSM9 [9,12]. However its precise role and involvement in signalling pathways remain elusive.

Arl13B belongs to the Arf (ADP-ribosylation factor) subfamily of the Ras superfamily of small G-proteins. They cycle between an inactive GDP-bound and an active GTP-bound conformation $[13,14]$. In the latter state they elicit cellular responses by binding to effector proteins, which are defined as binding specifically to the active state $[15,16]$. Since GTP hydrolysis and GDP/GTP exchange are usually intrinsically slow, the cycle is regulated by GAPs (GTPase-activating proteins) and GEFs (guaninenucleotide-exchange factors) enhancing intrinsic rates by orders of magnitude [17,18]. G-proteins of the Arf subfamily are structurally unique compared with other subfamilies of the Ras superfamily [19]. Their GDP/GTP transition shows an additional conformational switch called the interswitch toggle [20]. It is considered a communication device between the nucleotidebinding site and the amphipathic N-terminal helix present in all Arf/Arl proteins. In the inactive GDP-bound conformation, the N-terminal helix is bound in a hydrophobic pocket of the protein. Upon GTP binding the interswitch region shifts along the rest of the $\beta$-sheet by two residues such that the canonical interactions between switch I and switch II and the $\gamma$-phosphate become established [20,21]. In the course of this process, the $\mathrm{N}$ terminal helix is released from the pocket and becomes solvent(or membrane-) exposed. The structural dynamics and energetics of this remarkable conformational change are still to be explored.

Arl13B is set apart from other G-proteins of the Arf subfamily in that it contains, in addition to the $\mathrm{N}$-terminal G-domain, an extended C-terminus comprising a CC (coiled coil) and PRR (proline-rich region) [22]. A further major difference to other Arf family proteins is the absence of a catalytic glutamine/histidine residue in switch II. Although the nucleotide-binding ability of Arl13B has been described previously [6,22], the intrinsic GTPase activity for Arl13B has not been investigated up to date. Two missense mutations have been linked to Joubert syndrome [6], R79Q in the G-domain and R200C in the CC region. Since these mutations seem not to impair localization of Arl13B to cilia [23], the molecular basis of how they induce Joubert syndrome pathology continues to remain unclear. To further our understanding of the regulation of Arl13B GTPase activity and the effects of mutations, we used biochemical and high-resolution structural approaches to study Arl13B from Chlamydomonas rheinhardtii. Chlamydomonas is a unicellular green alga which

Abbreviations: Arf, ADP-ribosylation factor; Arl13B, ADP-ribosylation factor-like 13B; BART, binder of ARL2; CC, coiled coil; CrArl13B, Chlamydomonas rheinhardtii Arl13B; DTE, dithioerythritol; GAP, GTPase-activating protein; GEF, guanine-nucleotide-exchange factor; HRG4, human retinal gene 4; mant, $N$-methylanthraniloyl; $\mathrm{p}[\mathrm{NH}] \mathrm{ppG}$, guanosine $5^{\prime}$-[ $\beta$ - $\gamma$-imido]triphosphate; PRR, proline-rich region; RP2, retinitis pigmentosa 2; WT, wild type.

1 To whom correspondence should be addressed (email alfred.wittinghofer@mpi-dortmund.mpg.de).

The structural co-ordinates reported for C-terminally truncated Arl13B from Chlamydomonas rheinhardtii bound to p[NH]ppG will appear in the PDB under code $4 \mathrm{M} 9 \mathrm{Q}$. 
moves by the means of two flagella. Since its flagella also consist of a microtubular axoneme similar to the ciliary axoneme, Chlamydomonas is often used as a model system to investigate proteins localizing to cilia/flagella or regulating the traffic inside this compartment $[1,24]$.

In the present study we solved the structure of a construct of CrArl13B (Chlamydomonas rheinhardtii Arl13B) comprising the G-domain and part of the CC extension at $2.5 \AA(1 \AA$ $=0.1 \mathrm{~nm}$ ) resolution. The structure enabled us to study and interpret the position and effects of its mutations in human patients. We found that $\mathrm{Arg}^{200}$ and $\mathrm{Arg}^{79}$ stabilize the 3D structure through participation in intramolecular interactions as part of an interaction network of Arf/Arl proteins. We find $\mathrm{Arg}^{79}$ to be crucial for the conformational change of the interswitch toggle and that the Joubert mutation destabilizes the active state of the protein and is likely to cause a reduced affinity for its effectors.

\section{MATERIALS AND METHODS}

\section{Plasmids and protein purification}

CrArl13B (UniProt accession number A8INQ0) was amplified by PCR from a cDNA library from $C$. rheinhardtii CC-124 WT (wildtype) mt-[137c] [nit1, nit2, agg1] (a gift from Professor T. Happe, Department of Plant Biochemistry, Ruhr University Bochum, Bochum, Germany). In the present study shortened CrArl13B comprising amino acids 18-242 was used. The genes were cloned into pGexET (derivative of pGex4T-1) containing an Nterminal GST fusion followed by a thrombin, TEV (tobacco etch virus) and PreScission cleavage site (order as mentioned). Arl3 (UniProt accession number Q9WUL7) full length in pET20 was already available [25]. Respective CrArl13B ${ }^{\mathrm{R} 77 \mathrm{Q}}, \mathrm{CrArl13B}^{\mathrm{R} 194 \mathrm{C}}$ and $\mathrm{Arl} 3^{\mathrm{R} 75 \mathrm{Q}}$ mutants were generated by mutagenesis PCR. All proteins were expressed in Escherichia coli BL21 DE3 codon plus RIL cells at $25^{\circ} \mathrm{C}$ following induction with $100 \mu \mathrm{M}$ IPTG at $18^{\circ} \mathrm{C}$ overnight. Purification was done using GSH-Sepharose columns (GE Healthcare) which were washed with wash buffer [75 mM Hepes, pH 7.5, $300 \mathrm{mM} \mathrm{KCl,} \mathrm{5mM} \mathrm{MgCl}_{2}, 3 \mathrm{mM}$ 2-mercaptoethanol and $10 \%(\mathrm{v} / \mathrm{v})$ glycerol]. The GST-fusion proteins were eluted with elution buffer (wash buffer plus $20 \mathrm{mM}$ GSH). Following cleavage with PreScission protease, overnight residual GST was removed by size-exclusion chromatography using a Superdex 200 26/60 (GE Healthcare). Arl3 proteins containing a C-terminal His tag were purified as described previously [25,26]. The proteins were stored in buffer $\mathrm{M}$ containing $25 \mathrm{mM}$ Hepes, pH 7.5, $150 \mathrm{mM} \mathrm{KCl,} 5 \mathrm{mM} \mathrm{MgCl}_{2}$, $1 \mathrm{mM}$ DTE (dithioerythritol) and $5 \%$ (v/v) glycerol.

\section{Crystallization}

Native CrArl13B was purified according to the procedure mentioned above. The protein was exchanged as described previously $[25,26]$ to be either completely loaded with GDP, GTP or $\mathrm{p}[\mathrm{NH}] \mathrm{ppG}$ (guanosine $5^{\prime}$-[ $\beta$ - $\gamma$-imido $]$ triphosphate) and concentrated to approximately $14.8 \mathrm{mg} / \mathrm{ml}$. The sitting drop/vapour diffusion method was used and an initial condition was found in an identical condition in EasyXtal PEG II Suite and EasyXtal CLASSIC Suite from QIAGEN. The condition was optimized to a final solution of $0.2 \mathrm{M}$ ammonium sulfate, $0.1 \mathrm{M}$ Mes, pH 6.5, and $30 \%$ PEGMME [poly(ethylene glycol) monomethyl ether] 5000. Crystals of space group $P 2_{1} 2_{1} 2_{1}$ (Supplementary Table S1 at http://www.biochemj.org/bj/457/bj4570301add.htm) were reproducible and usually appeared after 1-3 days, dissolved and appeared again after 8 days. They were flash frozen after 9 days in a cryo-solution containing the same constituents as the crystallizing condition supplemented with $20 \%$ (v/v) glycerol. Data collection was done at the PXII-X10SA beamline of the Swiss Light Source (SLS), Villigen and P11 beamline at PETRA III at DESY campus, Hamburg, Germany. Data were indexed and processed with XDS [27]. Molecular replacement using different Arl structures was done with MOLREP and PHASER from the CCP4 package [28]. A model of the CrArl13B sequence generated by the PHYRE threader based on Arl2 (PDB code 1KSH [29]) was used successfully in molecular replacement to solve the CrArl13B structure. The structure was refined using REFMAC5 [30] to the following resolution (Ramachandran statistics in parentheses): CrArl13B native to $2.5 \AA$ (97.2\% favoured, $2.8 \%$ allowed, $0 \%$ outlier). For data and refinement statistics, see Supplementary Table S1. All the Figures were produced using PyMOL (http://www.pymol.org).

Atomic co-ordinates and structural factors have been deposited in the PDB under the accession code 4M9Q.

\section{Analytical size-exclusion chromatography}

The oligomerization state of Arl13B ${ }^{\mathrm{WT}}$ and mutant proteins was investigated by analytical size-exclusion chromatography using a Superdex 200 10/300 column (GE Healthcare). A total of $0.5 \mathrm{mg}$ of CrArl13B protein was incubated with a 10 -fold molar excess of GDP or $\mathrm{p}[\mathrm{NH}] \mathrm{ppG}$ for $2 \mathrm{~h}$ at room temperature $\left(25^{\circ} \mathrm{C}\right)$. The mix was then applied to the size-exclusion chromatography column and eluted with 1 column volume of buffer $\mathrm{M}$. The elution profile was recorded and eluted fractions were analysed by SDS/PAGE.

\section{Measurement of GTP hydrolysis by HPLC}

Briefly, $100 \mu \mathrm{M}$ GTP and $100 \mu \mathrm{M}$ CrArl13B ${ }^{\mathrm{WT}}-\mathrm{GTP}$ were incubated in buffer $\mathrm{M}$ at room temperature. Aliquots $(30 \mu \mathrm{l})$ were taken at $0,30,60,120,180,240$ and $360 \mathrm{~min}$. The protein was denatured by incubation for $5 \mathrm{~min}$ at $95^{\circ} \mathrm{C}$ and the nucleotide content of the supernatant was determined by HPLC measurements on a $\mathrm{C}_{18}$ column. GTP hydrolysis was also monitored under multiple turnover conditions by mixing $100 \mu \mathrm{M}$ GTP with $1 \mu \mathrm{M}$ CrArl13B ${ }^{\text {WT }}$. The mutant proteins R77Q and R194C were also measured under single and multiple turnover conditions.

\section{NMR experiments}

${ }^{31} \mathrm{P}$ and ${ }^{1} \mathrm{H}$ NMR spectra were recorded with a Bruker AVANCE-500 NMR spectrometer operating at a ${ }^{31} \mathrm{P}$ frequency of $202 \mathrm{MHz}$. Measurements were performed in a ${ }^{31} \mathrm{P}$ selective $10-\mathrm{mm}$ probe by using $8-\mathrm{mm}$ Shigemi sample tubes at various temperatures. CrArl13B proteins (approximately $1 \mathrm{mM}$ solutions) in $25 \mathrm{mM}$ Hepes/ $\mathrm{NaOH}, \mathrm{pH} 7.5,150 \mathrm{mM} \mathrm{KCl}$, $5 \mathrm{mM} \mathrm{MgCl} 2$ and $1 \mathrm{mM}$ DTE contained $10 \%{ }^{2} \mathrm{H}_{2} \mathrm{O}$ to get a lock signal. 2,2-Dimethyl-2-silapentane-5-sulfonate $(0.2 \mathrm{mM})$ was added to calibrate the spectra by indirect referencing according to Maurer and Kalbitzer [31]. Pulses of $70^{\circ}$ were applied with a total repetition time of $7 \mathrm{~s}$. Protons were decoupled by GARP sequence during data acquisition. The resonance assignment was transferred from Meierhofer et al. [32]. Additional 1D NOESY ${ }^{1} \mathrm{H}$ NMR spectra of the same samples were recorded on a Bruker AVANCE 600 NMR spectrometer at $278 \mathrm{~K}$.

\section{Affinity measurements}

$\mathrm{Arl} 3^{\mathrm{WT}}$ or mutant proteins were loaded with mant $(\mathrm{N}$ methylanthraniloyl)-GDP or mant-p[NH]ppG (Pharma Waldhof) overnight at $12{ }^{\circ} \mathrm{C}$ by incubation with a 1.5 -fold molar excess of nucleotide and purified the following day on a desalting 
A

\section{CrArl13B FL}



\section{CrArl13B}

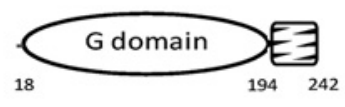

B
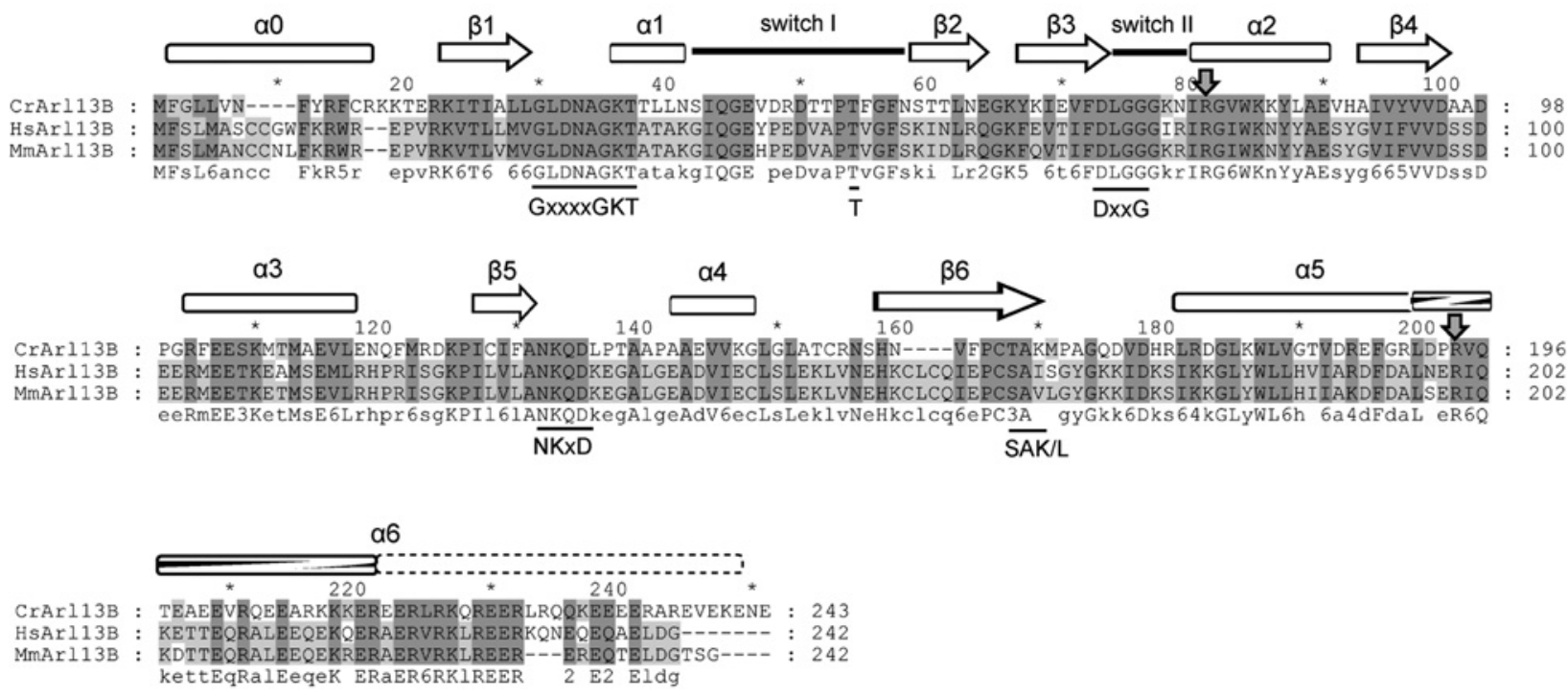

Figure 1 Domain organization and secondary structure of CrArl13B

(A) Domain organization of CrArl13B with amino acid boundaries of the G-domain, the CC and the PRR. The bottom panel shows the recombinant fragment (amino acids 18-242) used in the present study. (B) Alignment of residues 1-242 of Arl13B proteins from Homo sapiens (Hs) (UniProt accession number Q3SXY8), Mus musculus (Mm) (UniProt accession number Q640N2) and C. rheinhardtii (Cr) (UniProt accession number A8INQ0). Dependent on their degree of conservation, residues are highlighted in dark and light grey. The G1-G5 motifs characteristic for the G-domain, switch I and II, and the secondary structure elements of CrArl13B are shown below and above the alignment respectively. Black arrows indicate residues mutated in Joubert Syndrome. Secondary structure elements above the sequence are from the structure determined in the present study (except for the rest of $\alpha 6$; see text).

column in buffer $\mathrm{M}$ [25]. Nucleotide loading was determined by HPLC measurements on a $\mathrm{C}_{18}$ column. Polarization data was recorded with a Fluoromax-4 spectrophotometer (Jobin Yvon), with excitation and emission wavelengths of mant-labelled nucleotides at 366 and $450 \mathrm{~nm}$ respectively. Binding affinities of Arl3 WT and mutant proteins to HRG4 (human retinal gene 4), BART (binder of ARL2) and RP2 (retinitis pigmentosa 2) were measured by monitoring the polarization signal during titration of $1 \mu \mathrm{M}$ Arl3 loaded with the respective nucleotide with increasing amounts of $\mathrm{HRG} 4, \mathrm{BART}$ or RP 2 at $20^{\circ} \mathrm{C}$ in buffer M. The data points obtained were fitted to a first-order reaction using Grafit5 (Erithacus software) to obtain the dissociation constant $K_{\mathrm{d}}$.

\section{Determination of dissociation rates}

CrArl13B $(10 \mu \mathrm{M})$ was titrated with $1 \mu \mathrm{M}$ mant-GDP, mant$\mathrm{p}[\mathrm{NH}] \mathrm{ppG}$, mant-ADP or mant-ATP in buffer $\mathrm{M}$ at $20^{\circ} \mathrm{C}$. When the exchange reached equilibrium, reflected by a plateau in the polarization signal, the dissociation of mant-labelled nucleotide was induced by the addition of $400 \mu \mathrm{M}$ unlabelled nucleotide, GDP, $\mathrm{p}[\mathrm{NH}] \mathrm{ppG}$, ADP or ATP respectively. The exchange was followed by monitoring the polarization signal (for wavelength see above). Single exponential functions were fitted to the data using Grafit5 (Erithacus software) to obtain the $k_{\text {off }}$ values.

\section{Tryptophan fluorescence}

Fluorescence data was recorded with a Fluoromax-4 spectrometer (see above) with excitation and emission wavelengths of tryptophan at 290 and $340 \mathrm{~nm}$ respectively as reported previously [33]. CrArl13B $(1 \mu \mathrm{M}) \mathrm{WT}$ or mutant proteins were preloaded with GDP and their tryptophan fluorescence was monitored before and after the addition of either $10 \mu \mathrm{M}$ GDP or GTP at $20^{\circ} \mathrm{C}$ in buffer $\mathrm{M}$.

\section{RESULTS}

\section{Expression and characterization of Arl13B from C. reinhardtii}

Since human Arl13B turned out to be refractory to recombinant expression, we wondered whether we could identify and work with a Chlamydomonas homologue, since this organism is frequently used as a model system for ciliary biology 
[1,24]. Chlamydomonas CrArl13B, similar to the human protein, consists of a G-domain, a CC domain and a PRR (Figure 1A). The major difference between the two homologues is that the PRR is considerably (99 amino acids) longer in the case of CrArl13B (Supplementary Figure S1 at http:// www.biochemj.org/bj/457/bj4570301add.htm). On expression of full-length CrArl13B in E. coli, the protein was consistently C-terminally degraded to smaller more stable constructs, most likely due to the non-compactness of the PRR, for which no secondary structure elements can be predicted. We succeeded in the purification of a smaller stable construct comprising the Gdomain and part of the $\mathrm{CC}$ region ranging from amino acid 18 to 242, denoted CrArl13B (Figure 1). Within amino acids 1-242, Chlamydomonas and human Arl13B are 39\% identical $(57 \%$ similar) and are predicted to form similar secondary structure elements. They also contain the characteristic sequence motifs of the G-domain (Figure 1B).

The protein purified from $E$. coli contains equimolar amounts of bound nucleotide in a $20 \%$ GDP to $80 \%$ GTP ratio as determined by HPLC measurements, indicating that the protein is active and has a reasonably high affinity to guanine nucleotides (Figure 2A). To see whether the bound nucleotide is functional and can be exchanged in the absence of a GEF, exchange kinetics were monitored by fluorescence polarization measurements (Figure 2B). Addition of $10 \mu \mathrm{M} \mathrm{CrArl} 13 \mathrm{~B}$ to $1 \mu \mathrm{M}$ mant-labelled GDP or $\mathrm{p}[\mathrm{NH}] \mathrm{ppG}$ led to an increase in polarization indicating binding of the fluorescent guanine nucleotides. The rate of binding is identical as would be expected if the reaction is ratelimited by the release of bound nucleotide. The mant-labelled nucleotides bound to the protein could then be exchanged by addition of an excess of unlabelled nucleotide monitored as a decrease in polarization (Figure 2B). We observed a difference in the dissociation reaction, with mant $-\mathrm{p}[\mathrm{NH}] \mathrm{ppG}$ being released approximately 5-fold slower than mant-GDP. Since affinity in most cases is dictated by the dissociation rate [13], we can assume that binding affinities of the nucleoside triphosphate is approximately 5-fold higher than that of the diphosphate. No increase in fluorescence polarization was observed upon addition of $10 \mu \mathrm{M}$ CrArl13B to $1 \mu \mathrm{M}$ mant-labelled ADP or ATP nucleotides, confirming specificity of CrArl13B for guanine, but not adenine, nucleotides (Figure 2B).

Despite being able to purify small amounts of human Arl13B proteins of varying length and stability, we were not able to observe nucleotide bound to the purified protein nor could we detect any specific and, more importantly, reversible nucleotide exchange. The recombinant human protein bound unspecifically and irreversibly to all nucleotides tested suggesting a lack of proper folding (results not shown).

\section{Structure of Arl13B from C. rheinhardtii}

No crystals could be obtained for CrArl13B bound to GTP or GDP. However, CrArl13B bound to the non-hydrolysable GTP analogue $\mathrm{p}[\mathrm{NH}] \mathrm{ppG}$ crystallized in space group $P 2_{1} 2_{1} 2_{1}$ and diffracted to $2.5 \AA$ resolution (Supplementary Table S1). The asymmetric unit contained three Arl13B-p[NH]ppG monomers (Supplementary Figure S2 at http://www.biochemj.org/bj/457/bj4570301add.htm) in which the $\alpha 4$ and $\alpha 5$ helices and the $\beta 6$ strand of one monomer are contacting the $\alpha 2$ helix/switch II and the $\beta 2$ strand/switch I of a neighbouring monomer. Each monomer is turned by $45^{\circ}$ respective to the next monomer. Further packing produces a fibre-like structure of molecules in the crystal. To investigate the possibility that the packing is of any biochemical/physiological significance, we analysed a different crystal form. Crystals of space group $C 222_{1}$, which diffracted to approximately a


Figure 2 Nucleotide binding of CrArl13B

(A) HPLC measurement of nucleotide being bound to CrArl13B after purification. The protein/nucleotide ratio is $1: 1$, with a GDP/GTP ratio of $20 \%$ to $80 \%$. (B) Fluorescence polarization measurement at $20^{\circ} \mathrm{C}$ in buffer $\mathrm{M}$. To $1 \mu \mathrm{M}$ mant-labelled nucleotides (ADP, ATP, GDP or p[NH]ppG as indicated) was added $10 \mu \mathrm{M} \mathrm{CrArl13B}^{\mathrm{WT}}$ (first black arrow) and $400 \mu \mathrm{M}$ unlabelled nucleotide (second black arrow). GppNHp, p[NH]ppG.

$3.5 \AA$ resolution, contained an identical arrangement of three monomers per asymmetric unit (results not shown) which, however, showed no fibre formation as observed in $P 2_{1} 2_{1} 2_{1}$. Gel-filtration experiments show that, compared with gel filtration standards, CrArl13B elutes as a monomer in solution (see also below, Figure 3C).

CrArl13B contains a typical G-domain fold with a six-stranded $\beta$-sheet surrounded by five $\alpha$-helices (Figure 3A). The $\alpha 6$ helix, which is part of the CC region, emanates from the G-domain. For reasons not obvious from the sequence, it forms a sharp $90^{\circ}$ turn relative to the $\alpha 5$ helix of the G-domain and is apparently held in place by intramolecular interactions (Supplementary Figure S3A at http://www.biochemj.org/bj/457/bj4570301add.htm). The contact between the G-domain and the $\alpha 6$ helix is formed by two loops, one connecting helix $\alpha 2$ to $\beta 4$ and the other linking helix $\alpha 3$ to $\beta 5$. The loop linking $\alpha 3-\beta 5$ seems to form more contacts between the G-domain and the $\alpha 6$ helix, with $\operatorname{Arg}^{194}$ playing a central role (Supplementary Figure S3A, zoom). The loop connecting $\alpha 2-\beta 4$ is adjacent to $\alpha 2$ which in Arf/Arls belongs to switch II moving upon the GDP-GTP transition. The $\alpha 2-\beta 4$ loop itself, however, does not move upon the GDP-GTP transition as compared with other Arf/Arl structures. Despite the construct comprising amino acids 18-242, only amino acids 18-212 are 
A

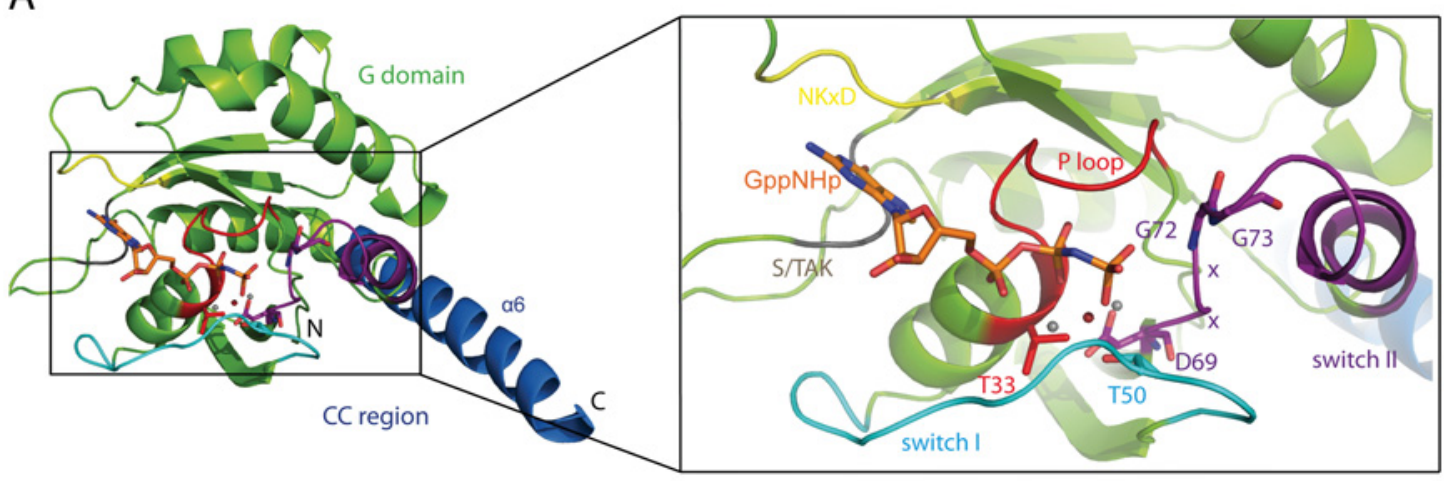

B


Figure 3 Structure of CrArl13B

(A) Overview of the CrArl13B structure (left-hand panel) and zoom (right-hand panel) of the active site, with CC region (blue), switch I (light blue), switch II (lilac) including the DxxG motif, P-loop (red), NKXD (yellow) and the S/TAK motif (dark grey). $\mathrm{Mg}^{2+}$ is co-ordinated by two water molecules (grey), the $\beta$ - and $\gamma$-phosphate of the nucleotide, Thr ${ }^{50}$ of switch I and Thr ${ }^{33}$ of the P-loop (red). (B) GTP hydrolysis measurement of either $100 \mu \mathrm{M}$ GTP, $100 \mu \mathrm{M}$ CrArl13B preloaded with GTP or $100 \mu \mathrm{M}$ GTP incubated with $1 \mu \mathrm{M}$ CrArl13B. (C) Analytical size-exclusion chromatography (Superdex 200 10/300GL) of CrArl13B'WT bound to GDP (light grey) and p[NH]ppG (dark grey). Gel filtration standards are shown in background: thyroglobulin $670 \mathrm{kDa}$ (1), $\gamma$-globulin $159 \mathrm{kDa}$ (2), ovalbumin $44 \mathrm{kDa}$ (3) and myoglobin $17 \mathrm{kDa}$ (4). GppNHp, p[NH]ppG.

visible in the electron density. Since the rest of the construct is predicted as an $\alpha$-helix and there is enough space in the crystal for the extension of $\alpha 6$, we suspect that the missing residues are not visible due to intrinsic mobility of the C-terminal end of the helix. This is further supported by the observation that in crystals of space group $C 222_{1}$, amino acids $18-224$ are now visible and that in both crystals helix $\alpha 6$ is pointing away in a straight line from the G-domain and is not involved in any crystal contacts, most likely adding to its flexibility. The residues in question are, however, present on the protein to be crystallized since no further degradation of CrArl13B was observed during purification and handling.

\section{The active site of CrArl13B lacks a catalytic glutamine residue}

A close look at the active site of CrArl13B shows that it contains all conserved residues required for guanine nucleotide binding in canonical orientation, such as the P-loop, a threonine residue in switch I, DxxG in switch II, and the NKxD and SAK motifs required for guanine specificity (Figures $1 \mathrm{~B}$ and $3 \mathrm{~A}$ ). The location

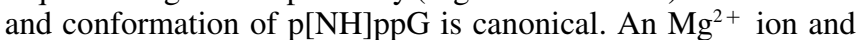
water molecules can be seen in the active site (Figure 3A). The $\mathrm{Mg}^{2+}$ is co-ordinated by the $\beta$ - and $\gamma$-phosphate of the nucleotide, the side-chain oxygens of $\mathrm{Thr}^{33}$ (P-loop) and $\mathrm{Thr}^{52}$ (switch I) and two water molecules.

Analysis of the sequence had suggested a failure of Arl13B to hydrolyse GTP, as glutamine, the residue following the consensus
DxxG (G4) motif, is replaced by glycine to give $\mathrm{D}^{69} \mathrm{xxGG}^{73}$ in Arl13B. Glutamine in that position is found in most other members of the Ras superfamily, including other Arl proteins (Figure 1B and Supplementary Figure S4 at http://www.biochemj. org/bj/457/bj4570301add.htm). This residue is crucial for GTP hydrolysis as it stabilizes the position of the nucleophilic water molecule. In the structure we find no other residue that could potentially be involved in catalysing phophoryl transfer. The absence of intrinsic GTP hydrolysis was confirmed by HPLC measurements under single and multiple turnover conditions (Figure 3B). In contrast with observations by Hori et al. [22], it can be excluded that a catalytic residue is inserted in trans into the active site from neighbouring monomers identified in the structure, suggesting that Arl13B might not be a GAD, a G-protein activated by dimerization [34]. GTP hydrolysis by dimerization of CrArl13B can probably be ruled out as the protein elutes as monomer of $25 \mathrm{kDa}$ in analytical gel filtration independently of the nature of the bound nucleotide (Figure 3C).

\section{The Joubert mutations Arg $^{77}$ and Arg $^{194}$}

Considering the expected close structural homology based on the overall sequence conservation, $\mathrm{CrArl13B}$ can most likely be used as a model to investigate the human patient mutations R79Q and R200C, which are fully conserved between organisms as indicated in Figure 1(B) (Supplementary Figure S1). Human residues Arg ${ }^{79}$ and $\mathrm{Arg}^{200}$ correspond to $\mathrm{Arg}^{77}$ and $\mathrm{Arg}^{194}$ in the Chlamydomonas 
A

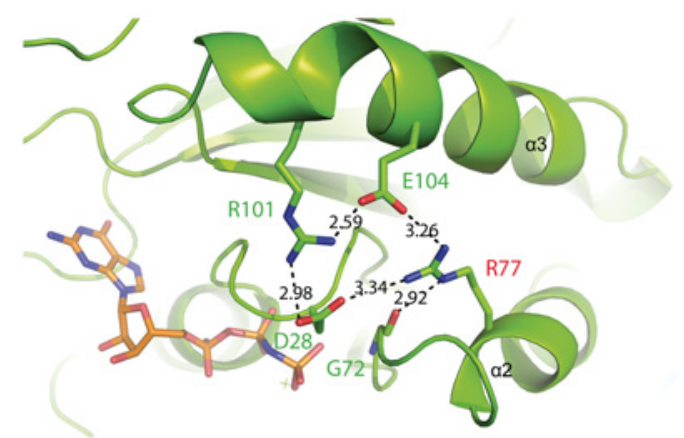

B

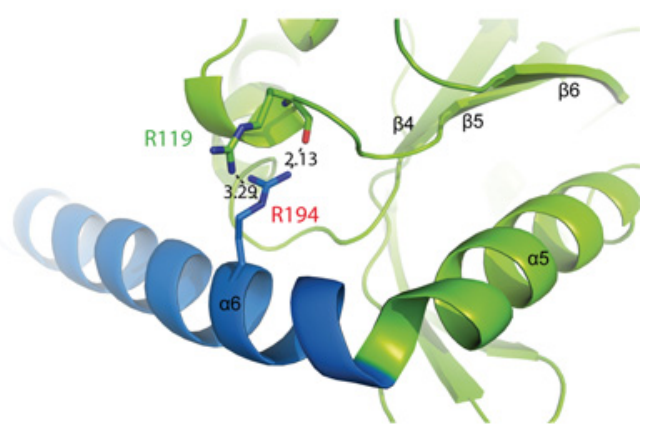

GppNHp



Figure 4 Patients with Joubert syndrome mutations

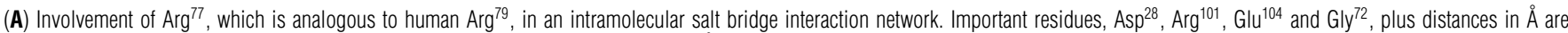

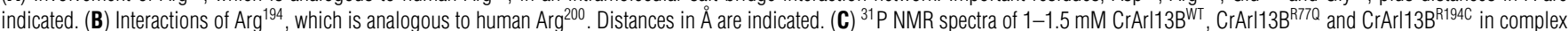
with $\mathrm{Mg}^{2+} . p[\mathrm{NH}] \mathrm{ppG}$ at $278 \mathrm{~K}$ in buffer M. Signals are assigned according to Meierhofer et al. [32]. GppNHp, p[NH]ppG.

protein. $\operatorname{Arg}^{77}$ is located on the $\alpha 2$ helix of the G-domain and is orientated towards the interior (Figure 4A and Supplementary Figure S3B). It forms a salt bridge network with $\mathrm{Asp}^{28}$ of the P-loop and $\mathrm{Arg}^{101}$ and $\mathrm{Glu}^{104}$ of the $\alpha 3$ helix. Considering the large conformational change in the GDP-GTP transition of the switch II region of all Arf and Arl proteins [19,20], it appears that these intramolecular salt bridges stabilize particularly the GTP-bound protein conformation and the position of switch II (Supplementary Figure S3B). The bridging nitrogen $(\mathrm{N} \varepsilon)$ of $\mathrm{Arg}^{77}$ further establishes a hydrogen bond to the backbone oxygen of $\mathrm{Gly}^{72}$ of the DxxG motif. This glycine residue also forms the canonical $\mathrm{NH}$ main chain interaction with the $\gamma$-phosphate (Supplementary Figure S3B). Arg $^{194}$ is located within the CC region on the $\alpha 6$ helix (Figure $4 \mathrm{~B}$ ). The guanidinium group of $\mathrm{Arg}^{194}$ forms a hydrogen bond to the main chain oxygen of $\operatorname{Arg}^{119}$ via the terminal nitrogen. There is an additional salt bridge between $\mathrm{N} \omega$ of the $\mathrm{Arg}^{119}$ guanidinium group and the bridging nitrogen $\mathrm{N} \varepsilon$ of the guanidinium group of $\mathrm{Arg}^{194}$. These interactions appear to stabilize the position of the $\alpha 6$ helix relative to the G-domain, which makes a sharp $90^{\circ}$ turn relative to helix $\alpha 5$ (see above, Supplementary Figure S3A).

$\mathrm{Arg}^{77}$ and $\mathrm{Arg}^{194}$ mutations to glutamine and cysteine respectively, as found in patients with Joubert syndrome in analogous positions, are expected to disrupt the intramolecular interactions. We thus introduced the R77Q and R194C substitutions into CrArl13B and could purify soluble functional proteins in both cases (Supplementary Figures S5C and S5D at http://www.biochemj.org/ bj/457/bj4570301add.htm). Similarly to the WT protein, both mutants lack GTP hydrolysis (Supplementary Figure S5B). ${ }^{31} \mathrm{P}$ -
NMR spectroscopy using the bound nucleotide as a probe is a sensitive method to detect structural changes in the active centre of Ras superfamily proteins [35]. The most dominant factors determining the obtained ${ }^{31} \mathrm{P}$ chemical shifts are the conformational strain and electric field effects polarizing the phosphate oxygens of the nucleotide. Long-range effects due to structural rearrangement can also lead to changes in the anisotropy of the magnetic susceptibility, e.g. ring current effects of aromatic side chains. Although chemical shift changes cannot be interpreted in a simple way, the local chemical environment of the observed nucleus must be different. The ${ }^{31} \mathrm{P}$ NMR spectrum of $\mathrm{p}[\mathrm{NH}] \mathrm{ppG}$ bound to WT CrArl13B shows three single signals corresponding to three phosphate groups (Figure 4C). The analysis of $\mathrm{p}[\mathrm{NH}] \mathrm{ppG}$ bound to $\mathrm{CrArl13B}$ mutants reveals changes in the observed chemical shift values or line-width for the mutant proteins compared with the WT (Supplementary Table S2 at http://www.biochemj.org/bj/457/bj4570301add.htm). For R77Q, a down-field shift of the $\beta$-phosphate signal from -0.45 p.p.m. to -0.36 p.p.m. and an up-field shift for the $\gamma$ phosphate signal from -3.06 p.p.m. to -3.42 p.p.m. are detected (Figure 4C and Supplementary Table S2). This observation implies a different chemical environment of the phosphate groups, probably linked to the inability to properly fix switch II in an active position. In contrast, no change in chemical shift can be seen for R194C compared with the WT spectrum, but the signals exhibit a significantly smaller line-width. The total linewidths at half height for the phosphate group signals in R194C mutant are 92, 82 and $62 \mathrm{~Hz}$ for the $\alpha-, \beta$ - and $\gamma$-phosphorous respectively, compared with 169,126 and $106 \mathrm{~Hz}$ obtained for WT (Supplementary Table S2). Additional line broadening in these 


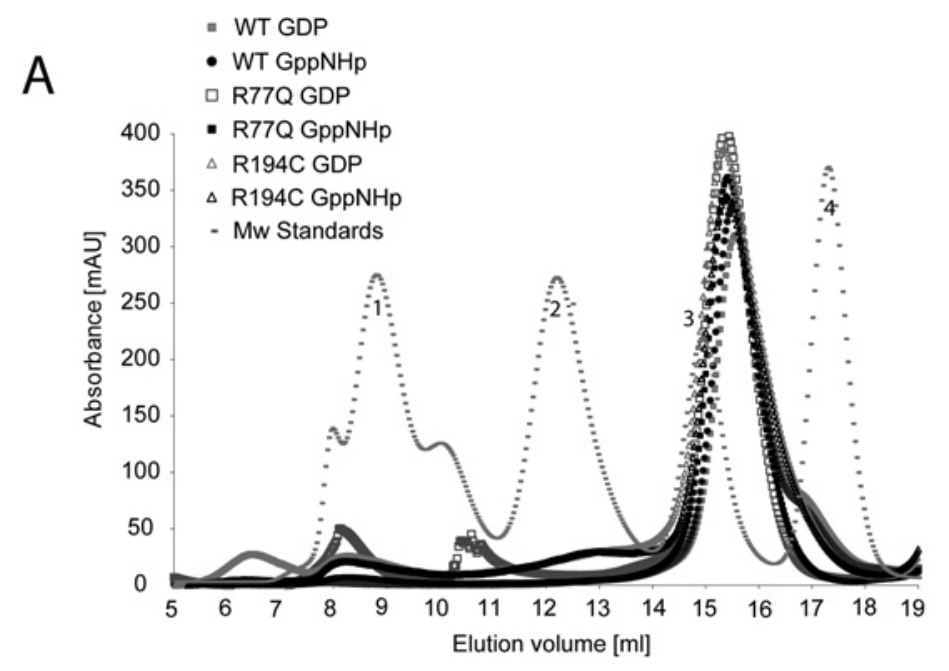

B
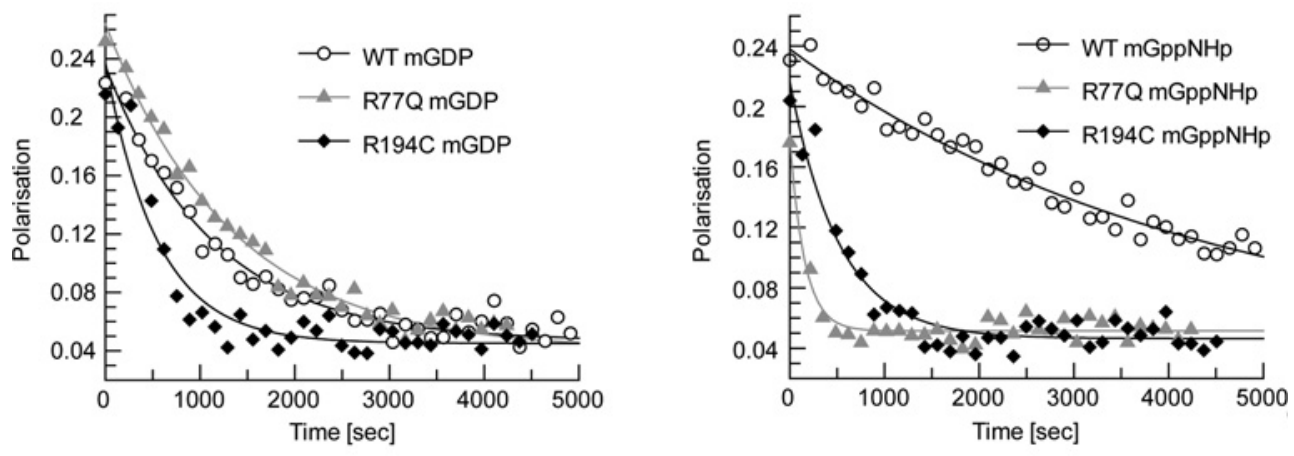

\begin{tabular}{l|lll} 
koff $\left[\mathrm{sec}^{-1}\right]$ & WT & R77Q & R194C \\
\hline mGDP & 0.0009 & 0.0008 & 0.0018 \\
mGppNHp & 0.0002 & 0.0063 & 0.0019
\end{tabular}

\section{Figure 5 Biochemical characterization of patient mutations}

(A) Analytical size-exclusion chromatography (Superdex 200 10/300GL) of WT or mutant CrArl13B bound to GDP or p[NH]ppG as indicated. Gel filtration standards are shown in background: thyroglobulin $670 \mathrm{kDa}$ (1), $\gamma$-globulin $159 \mathrm{kDa}$ (2), ovalbumin $44 \mathrm{kDa}$ (3) and myoglobin $17 \mathrm{kDa}$ (4). (B) Fluorescence polarization measurement of nucleotide dissociation at $20^{\circ} \mathrm{C}$ in buffer $\mathrm{M}$ of $1 \mu \mathrm{M}$ WT or mutant CrArl13B bound to either mant-GDP (mGDP) (left-hand panel) or mant-p[NH]ppG (mGppNHp) (right-hand panel) as indicated, initiated by the addition of $400 \mu \mathrm{M}$ unlabelled nucleotide. The curves were fitted to single exponentials in order to determine the $k_{\text {off }}$ rates which are listed in the table underneath the graphs.

experiments could principally be due to dimerization, leading to an increase of the rotational correlation time of the complex or the result of chemical exchange processes. Since the ${ }^{1} \mathrm{H}$ NMR data of the same inactive and active Arl13B complexes did not show any indications for dimerization (results not shown), these results can be explained by conformational exchange processes in the WT which are not observed in the R194C mutant or, if they exist, then exchange should exhibit much higher interconversion rates. Apart from some differences in line-width, which again indicates a chemical exchange process in the R77Q mutant, no chemical shift changes could be detected for GDP bound to the proteins, which suggests that these mutational changes have much more specific effects for the active conformation of CrArl13B (Supplementary Figure S5A).

Analytical gel filtration shows both R77Q and R194C eluting as monomers of approximately $25 \mathrm{kDa}$ independently of their nucleotide state (Figure 5A). Nucleotide affinities could not be determined as the nucleotide-free protein precipitated. As an indirect measure of affinities, dissociation rates $\left(k_{\text {off }}\right)$ were

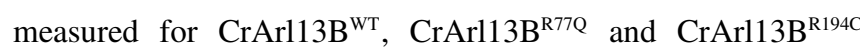
bound to either mant-GDP or mant-p[NH]ppG (Figure $5 \mathrm{~B}$ ). The exchange of the mant-labelled nucleotide to unlabelled nucleotide was followed by the change in fluorescence polarization over time. The $k_{\text {off }}$ values were determined by fitting a single exponential function to the curves. No major difference can be detected in the dissociation rates for GDP between WT and the mutants R77Q and R194C (Figure 5B, left-hand panel and table). In contrast, the $k_{\text {off }}$ value obtained for $\mathrm{p}[\mathrm{NH}] \mathrm{ppG}$ is increased by approximately 30-fold for R77Q (Figure 5B, right-hand panel and table) and 10-fold for R194C. This suggests that the structural changes are transmitted to the GTP-binding site in Joubert syndrome mutants.

\section{Arg $^{77}$ is essential for the stability of the GTP-bound conformation and interaction with effectors}

A DALI search shows Arl2, Arl3 and Arf1 to be the closest structural homologues of CrArl13B. An overlay of CrArl13B with 


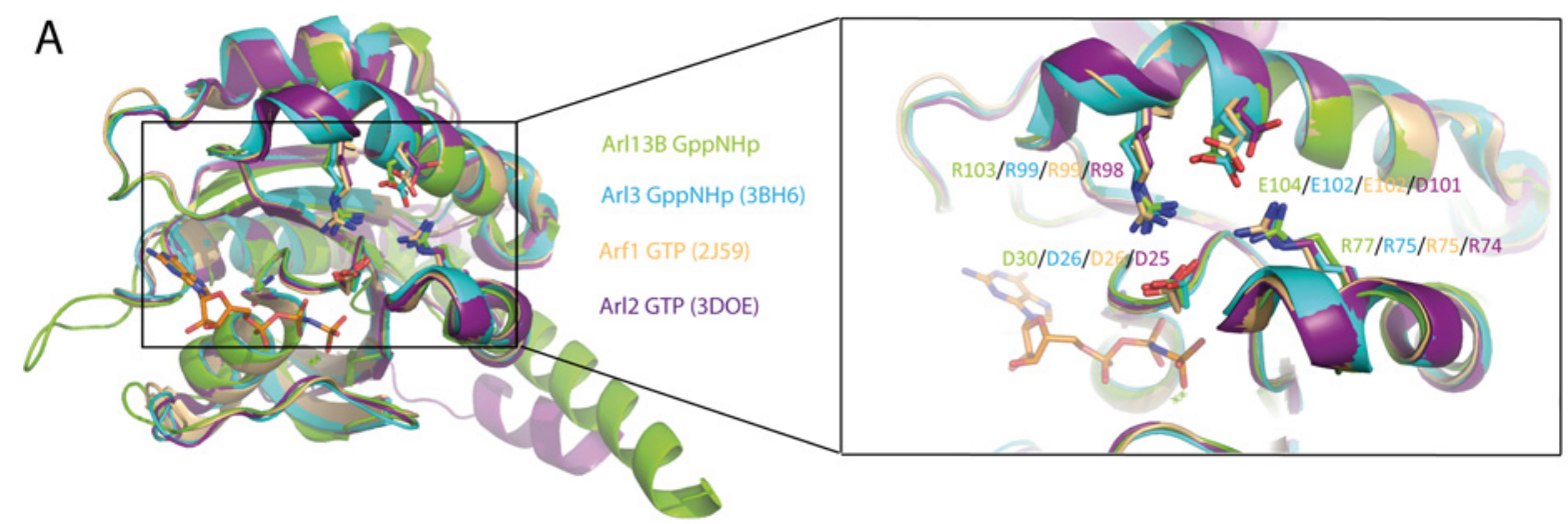

B
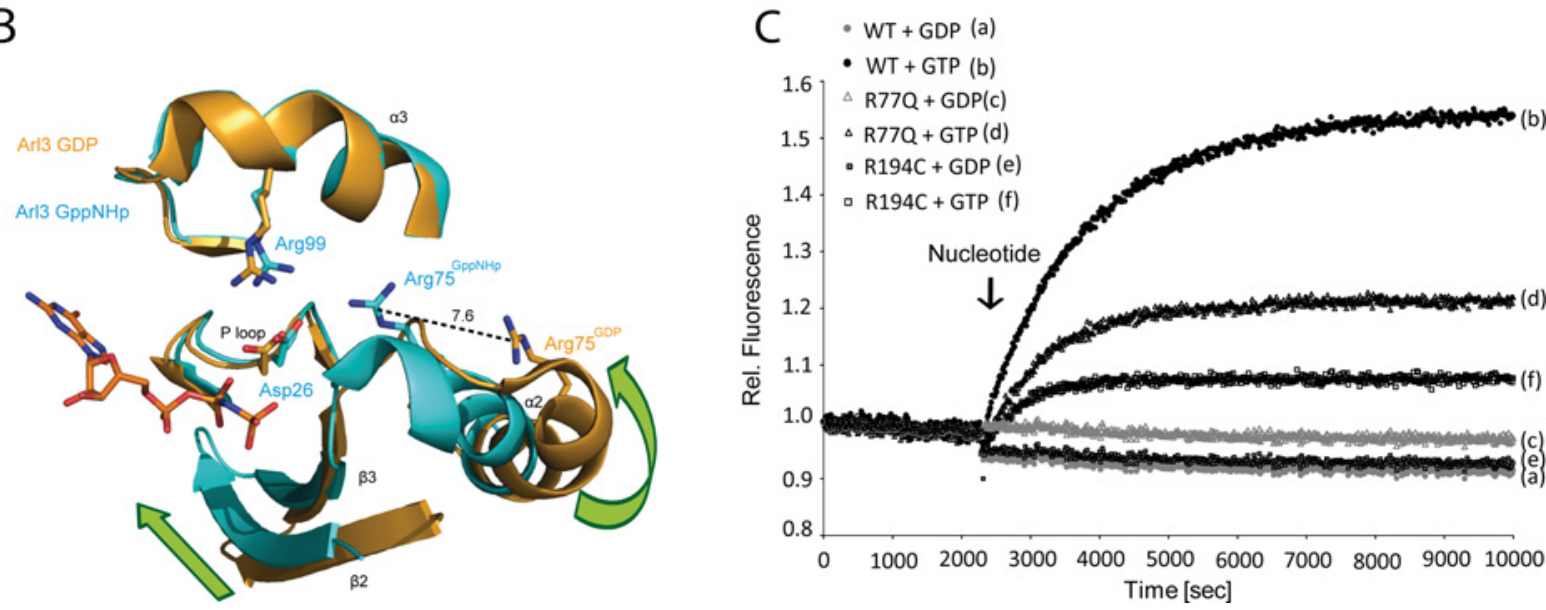

Figure 6 Comparison of Arl13B to other Arl/Arf subfamily members

(A) Overlay of CrArl13B-p[NH]ppG with Arl3-p[NH]ppG (PDB code 3BH6 [39]), Arf1-GTP (PDB code 2J59 [48]) and Arl2-GTP (PDB code 3D0E [44]) is shown on the left-hand side, the zoom into switch II on the right-hand side. The salt bridge interaction network between aspartate (P-loop), arginine ( $\alpha 3$ helix) and arginine ( $\alpha 2$ helix) residues can be found in all Arl/Arf structures. (B) Superimposition of Arl3-GDP (PDB code 1FZQ, orange [38]) on to Arl3-p[NH]ppG (PDB code 3BH6, cyan [39]) shows the conformational change of Arg ${ }^{75}$ and the $\alpha 2$ helix as part of switch II upon the GDP/GTP transition. (C) Tryptophan fluorescence monitored at $340 \mathrm{~nm}$ of $1 \mu \mathrm{M}$ WT or mutant CrArl13B-GDP following addition of $10 \mu \mathrm{M}$ GDP or GTP respectively in buffer M at $20^{\circ} \mathrm{C}$.

the structures of Arl3, Arl2 and Arf1 in their active conformation displays the high structural similarity of the proteins in the GTPbound state (Figure 6A, left-hand panel). The overlay shows that the salt bridge interaction network of $\mathrm{Arg}^{77}$ in $\alpha 2, \mathrm{Asp}^{28}$ in the P-loop and $\mathrm{Arg}^{101}$ in $\alpha 3$ of Arl13B might be important, since their positions are occupied by identical amino acids in all other available Arl and Arf structures (Figure 6A, right-hand panel and Supplementary Figure S4). Asp ${ }^{28}$ in the P-loop corresponds to residue $\mathrm{Gly}^{12}$ in Ras, where every mutation of Gly ${ }^{12}$ including aspartate blocks GAP-mediated GTP hydrolysis and makes Ras an oncogene [36]. Apparently the fixation of the aspartate residue away from the active site by the salt bridge network allows the ArfGAPs to insert its arginine finger and function properly [37]. Only Glu ${ }^{104}$ in $\alpha 3$ of CrArl13B is replaced by different amino acids in other Arf/Arl proteins. Alignment of a few representative G-proteins of each subfamily showed that the aspartate residue of the P-loop, the arginine residue in $\alpha 2$ and the arginine residue in $\alpha 3$ are highly conserved only in the Arf subfamily except Arl8 (Supplementary Figure S4). Hence, the three residues and their interactions seem to constitute a critical part of the Arf subfamily function.

In order to better understand the role of $\mathrm{Arg}^{77}$ in the GDP/GTP structural transition, we superimposed structures of Arl3-GDP [38] with Arl3-p[NH]ppG [39] (Figure 6B). Arl3 was chosen since no crystals could be obtained for CrArl13B-GDP and no structure of Arl2-GDP is available in the PDB. $\operatorname{Arg}^{75}$ in Arl3 is the residue corresponding to $\mathrm{Arg}^{77}$ in $\mathrm{CrArl13B}$. The overlay shows that, upon the GDP/GTP structural transition, the $\alpha 2$ helix as part of switch II undergoes a major conformational change such that the guanidinium group of $\mathrm{Arg}^{75}$ undergoes a movement of approximately $7.6 \AA$ (Figure 6B). Similar large conformational changes of switch II are documented by e.g. the structures of Arl3-p[NH]ppG (PDB code 3BH6 [39]) and Arf6-GTP (PDB code 2J5X [21]) compared with their GDP-bound structures (PDB codes 1FZQ [38] and 1E0S [33] respectively). Intriguingly, the $\alpha 2$ helix is connected to $\beta 3$, which together with $\beta 2$ forms what is called the interswitch toggle of Arf and Arl proteins. Tilting of $\alpha 2$ towards the active site consequently seems to push down the interswitch toggle. Movement of the interswitch toggle away from the active site usually is linked to the ejection of the $\mathrm{N}$-terminal helix.

This large conformational change as a result of the GDP/GTP transition can be monitored by a similar large change in tryptophan fluorescence as shown for other Arf proteins. A tryptophan residue in helix $\alpha 2$ of switch II was shown to change its conformation and is even more excluded from solvent upon the GDP/GTP structural transition of Arf proteins [20,33]. CrArl13B also possesses a tryptophan residue in switch II, apart from a second tryptophan residue in helix $\alpha 5$. WT and mutant CrArl13B were preloaded with GDP. The addition of GTP leads to a considerable 1.6fold increase in tryptophan fluorescence for the WT (Figure 6C), suggesting the same toggle movement characteristic for the Arf subfamily. The Joubert syndrome mutations R77Q and 
A

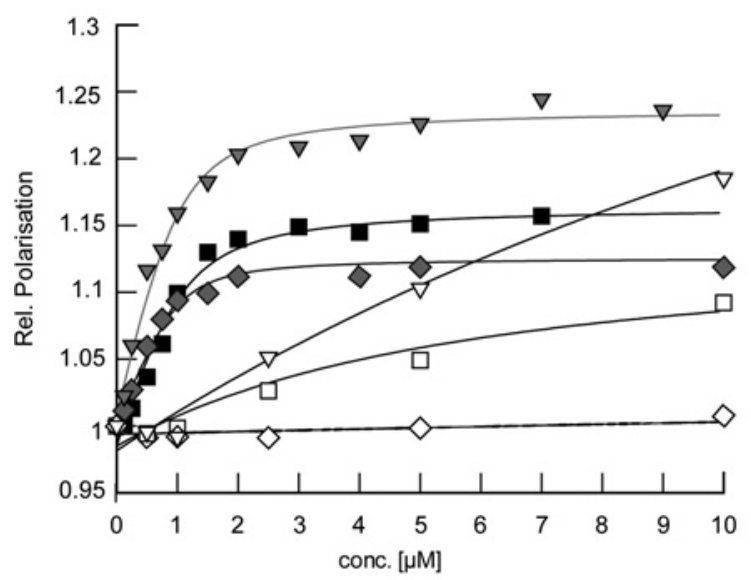

B $\because-1 \mu \mathrm{M}$ Arl3FL WT mGppNHp + HRG4

$-\square-1 \mu \mathrm{M}$ Arl3FL R75Q mGppNHp + HRG4

$\smile 1 \mu \mathrm{M}$ Arl3FL WT mGppNHp + BART

.. $\diamond . .1 \mu \mathrm{M}$ Arl3 R75Q mGppNHp + BART

$\rightarrow-1 \mu \mathrm{M}$ Arl3FL WT mGppNHp + RP2

$\rightarrow-1 \mu \mathrm{M}$ Arl3FL R75Q mGppNHp + RP2

\begin{tabular}{l|rr}
${\mathrm{Kd}[\mu \mathrm{M}]^{-1}}^{-1}$ WT & R75Q \\
\hline HRG4 & 0.250 & 5.1 \\
BART & 0.122 & 104.8 \\
RP2 & 0.184 & 21.5
\end{tabular}

\begin{tabular}{l|ll} 
koff $\left[\mathrm{sec}^{-1}\right]$ & WT & R75Q \\
\hline mGDP & 0.000004 & 0.000004 \\
mGppNHp & 0.0002 & 0.0016
\end{tabular}

Figure 7 Mutation of Arl3 ${ }^{\mathrm{R} 750}$

(A) Measurement of fluorescence polarization during titration of HRG4 or BART to full-length $1 \mu \mathrm{M}$ Arl $3^{\mathrm{WT}}$ or Arl $3^{\mathrm{R} 750}$ loaded with mant-p [NH]ppG (mGppNHp) at $20^{\circ} \mathrm{C}$ in buffer M as indicated. Fitting to a quadratic equation gives the dissociation constants shown in the table to the right-hand side. (B) Exchange rates of Arl $3^{\mathrm{R} 750}$ and Arl $3^{\mathrm{WT}}$ proteins, measured by fluorescence polarization as in Figure 5(B). mGDP, mant-GDP.

R194C show a decreased amplitude of tryptophan fluorescence change, only 1.2- or 1.1-fold respectively upon addition of GTP (Figure 6C). This suggests, as expected, that although the mutants do bind GTP or GTP analogues, the transition to and/or the stability of the active conformation is impaired in these mutants.

Considering the large conformational change in the switch region observed for Arf/Arl proteins and its stabilization by the salt bridge network, we speculated that mutation of $\mathrm{Arg}^{77}$ in Arl13B will result in impaired effector binding and N-terminal helix release. In the absence of any clearly defined Arl13B effector, we investigated the interaction of Arl3 and the corresponding R75Q mutant with the two effectors HRG4/Unc119a and BART by fluorescence polarization. Determination of the dissociation constant $\left(K_{\mathrm{d}}\right)$ for interaction of $\mathrm{Arl} 3^{\mathrm{WT}}$ and $\mathrm{Arl} 3^{\mathrm{R} 75 \mathrm{Q}}$ with the effectors shows that binding is dramatically impaired. HRG4/Unc119 binds to switch I and II and BART contacts the N-terminal helix in addition to the switch regions of Arl (Supplementary Figure S6 at http://www.biochemj.org/bj/457/bj4570301add.htm). In both cases the affinity is reduced by approximately 10 - to 1000 -fold for R75Q compared with WT (Figure 7A). To further confirm the analogy between Arl3 and Arl13B and the Joubert syndrome mutation $\mathrm{R} 77 \mathrm{Q}$, the $k_{\text {off }}$ value for $\mathrm{Arl3}{ }^{\mathrm{R} 75 \mathrm{Q}}$ bound to $\mathrm{p}[\mathrm{NH}] \mathrm{ppG}$ also increases, whereas it has no effect on GDP dissociation (Figure 7B). Furthermore, the Arl3-specific GAP RP2 also binds with approximately 100 -fold reduced affinity to $\mathrm{Arl} 3^{\mathrm{R} 75 \mathrm{Q}}$ (Figure 7A) with consequently impaired GTP hydrolysis (results not shown). Thus these data suggest that the ability to adopt the active conformation is severely affected by this mutation.

\section{DISCUSSION}

The Arf/Arl subfamily sets itself apart within the Ras superfamily in possessing an interswitch toggle linked to an N-terminal amphipathic helix, which in many members is lipid modified and thought to establish membrane interactions [19]. The structural determinants of that extremely large conformational change remain to be explored. Apart from Arl3 and Arl6, Arl13B is one of the three Arl proteins found in the proteome of ciliated organisms. Both Arl6 [40,41] and Arl13B [6,8,42] have been reported to be mutated in human disease, causing the ciliopathies Bardet Biedl Syndrome and Joubert Syndrome respectively. Knockout of Arl3 in mice causes severe defects in the function of photoreceptors. Whereas the role of Arl3 in ciliary trafficking of prenylated and myristoylated proteins is beginning to be explored [43], the role of Arl13B and the regulation and function of its GTPase cycle in cilia is unknown. In light of this the consequences of the described disease mutations of Arl proteins in ciliopathies need to be investigated.

The present study aimed to understand the mechanistic consequences of the Ar113B mutations R79Q and R200C that cause Joubert syndrome [6]. We are rather confident that the results from Chlamydomonas can be extrapolated to the human protein. We thus show that the residues analogous to the mutations R79Q and R200C are structurally important for the functional integrity of the Arl13B G-domain. Arg ${ }^{200}$ seems to link the long $\alpha 6$ helix of the $\mathrm{CC}$ region to the $\mathrm{G}$-domain. We speculate that the R200C mutation destabilizes the position of helix $\alpha 6$ of the CC region relative to the $\mathrm{G}$-domain. It suggests that a fixed orientation of the $\mathrm{CC}$ region is crucial for the function of Arl13B. On the basis of the structure of $\mathrm{CrArl13B}$ we identified $\mathrm{Arg}^{79}$ to be critical for establishing the proper GTP-bound conformation of Arl13B needed for effector binding and interaction with a GAP protein. $\mathrm{Arg}^{79}$ participates in an extended salt bridge network in the active state. We find this interaction network involving $\operatorname{Arg}^{79}$ to be a characteristic of the entire Arf/Arl subfamily. Previously it has been suggested that wDvGGqxxxRxxW (G3 motif, switch II) is a signature sequence for Arl proteins that toggle [20], however the 
importance of this arginine residue has not been investigated. Comparison of the GDP- and GTP-bound structures of Arl3 (and others) shows that this arginine residue undergoes a drastic translocation upon activation.

Using Arl3 as example, we demonstrate that mutation of the arginine residue to glutamine indeed impairs effector binding as well as binding of Arl3 to its GAP RP2. Since small G-proteins require an intact switch II region for interaction with effectors and assuming that as in $\mathrm{Arl} 3$ the $\mathrm{Arg}^{77}$ mutation disturbs the interaction with its cognate GAP, we may assume that the Joubert syndrome mutants are mostly GTP-bound. Nevertheless these mutants are loss-of-function mutants due to the lack of effector binding. In case of Arl3, an effector interaction, e.g. with BART involving the N-terminal helix of Arl3 additionally to its switches [44] seems even more severely affected by this mutation. Therefore the inability to stabilize switch II appears to disable the efficient release of the N-terminal helix upon the GDP/GTP transition. The GDP nucleotide-exchange reaction for WT and mutant Arl13B are similarly slow with a half-life in the order of 6-12 min, which suggests that Arl13B might require a GEF for loading with GTP.

Arl13B ${ }^{\mathrm{R} 79 \mathrm{Q}}$ becomes localized to cilia in the same way as the WT protein [23]. Therefore we can conclude that transport of Arl13B to cilia might take place independently of its GTPase activity, i.e. the hydrolysis of GTP and/or binding to an upstream regulator or downstream effector. Considering that GTP binding to $\operatorname{Arl} 13 \mathrm{~B}^{\mathrm{R} 200 \mathrm{C}}$ is also weakened as in the R79Q mutant, we would assume that this mutation, which probably destabilizes the position of the long CC helix, might be similarly affected in its ability to bind the effector and/or GAP. R200C localizes normally to cilia [23], but as R79Q it cannot rescue the ciliogenesis defect caused by Arl13B ${ }^{\mathrm{WT}}$ deletion. In the present study it has been demonstrated that the $\mathrm{CC}$ region and the PRR, which are $\mathrm{C}$-terminal to $\alpha 6$, are required, in addition to the potential palmitoylation $[9,42]$ of the $\mathrm{N}$-terminus, to be targeted to the cilium $[10,22,42]$.

The question of what function the GTP hydrolysis of Arl13B actually fulfils depends on the future identification of its regulators. The absence of a catalytic residue in the active site of Arl13B and the lack of dimerization for a construct comprising the $\mathrm{G}$-domain and part of the $\mathrm{CC}$ region suggest that regulation of Arl13B GTPase activity indeed is in need of a GAP, supposedly one that inserts an asparagine or a glutamine residue into the active site as shown for RapGAP [45,46] and RabGAP [47]. It is tempting to speculate that GTP hydrolysis might be linked to a conformational change extending to its $\mathrm{C}$-terminal $\mathrm{CC}$ and PRR or the regulation of IFT (intraflagellar transport). The latter is suggested by various reports of IFT-B and IFT-A dissociation upon Arl13B deletion [9,42].

\section{AUTHOR CONTRIBUTION}

Protein preparation, biochemical/biophysical measurements, crystallization, X-ray data analysis and paper preparation were done by Mandy Miertzschke. Carolin Koerner cloned various constructs and helped to purify proteins. NMR measurements and analysis were carried out by Michael Spoerner. Project design, supervision and paper preparation were supervised by Alfred Wittinghofer.

\section{ACKNOWLEDGEMENTS}

We thank Bernd Gilsbach, Mihai Gazdag, David Bier, Dominic Meusch and the SLS beamline staff for data collection of the crystals at the Swiss Light Source, beamline PXII X10SA, Paul Scherer Institute, Villigen, Switzerland and Ingrid Vetter, Eckhardt Hofmann and Arthur Porfetye and PETRA beamline staff Anja Burkhardt and Alke Meents for data collection of crystals at the P11 beamline at PETRA III synchrotron on the DESY campus in Hamburg, Germany. We thank Hans Robert Kalbitzer for the possibility to record ${ }^{31} \mathrm{P}$ NMR data at the University of Regensburg.

\section{FUNDING}

This work was supported by the European Research Council (ERC) Advanced Grant [Project Title: ARCID; number 268782].

\section{REFERENCES}

1 Fisch, C. and Dupuis-Williams, P. (2011) Ultrastructure of cilia and flagella - back to the future! Biol. Cell 103, 249-270

2 Goetz, S. C. and Anderson, K. V. (2010) The primary cilium: a signalling centre during vertebrate development. Nat. Rev. Genet. 11, 331-344

3 Eggenschwiler, J. T. and Anderson, K. V. (2007) Cilia and developmental signaling. Annu. Rev. Cell Biol. 23, 345-373

4 Badano, J. L., Mitsuma, N., Beales, P. L. and Katsanis, N. (2006) The ciliopathies: an emerging class of human genetic disorders. Annu. Rev. Genomics Hum. Genet. 7, $125-148$

5 Novarino, G., Akizu, N. and Gleeson, J. G. (2011) Review modeling human disease in humans: the ciliopathies. Cell 147, 70-79

6 Cantagrel, V., Silhavy, J. L., Bielas, S. L., Swistun, D., Marsh, S. E., Bertrand, J. Y., Audollent, S., Attié-Bitach, T., Holden, K. R., Dobyns, W. B. et al. (2008) Mutations in the cilia gene ARL13B lead to the classical form of Joubert syndrome. Am. J. Hum. Genet. 83, 170-179

7 Juric-Sekhar, G., Adkins, J., Doherty, D. and Hevner, R. F. (2012) Joubert syndrome: brain and spinal cord malformations in genotyped cases and implications for neurodevelopmental functions of primary cilia. Acta Neuropathol. 123, 695-709

8 Caspary, T., Larkins, C. E. and Anderson, K. V. (2007) The graded response to Sonic Hedgehog depends on cilia architecture. Dev. Cell 12, 767-778

9 Cevik, S., Hori, Y., Kaplan, O. I., Kida, K., Toivenon, T., Foley-Fisher, C., Cottell, D., Katada, T., Kontani, K. and Blacque, O. E. (2010) Joubert syndrome Arl13b functions at ciliary membranes and stabilizes protein transport in Caenorhabditis elegans. J. Cell Biol. 188, 953-969

10 Duldulao, N. A., Lee, S. and Sun, Z. (2009) Cilia localization is essential for in vivo functions of the Joubert syndrome protein Arl13b/Scorpion. Development 136, 4033-4042

11 Sun, Z., Amsterdam, A., Pazour, G. J., Cole, D. G. and Miller, M. S. (2004) A genetic screen in zebrafish identifies cilia genes as a principal cause of cystic kidney. Development 131, 4085-4093

12 Li, Y., Zhang, Q., Wei, Q., Zhang, Y., Ling, K. and Hu, J. (2012) SUMOylation of the small GTPase ARL-13 promotes ciliary targeting of sensory receptors. J. Cell Biol. 199, 589-598

13 Vetter, I. R. and Wittinghofer, A. (2001) The guanine nucleotide-binding switch in three dimensions. Science 294, 1299-1304

14 Cox, A. D. and Der, C. J. (2010) Ras history. Small GTPases 1, 1-27

15 Wittinghofer, A. and Nassar, N. (1996) How Ras-related proteins talk to their effectors. Trends Biochem. Sci. 21, 488-491

16 Herrmann, C. (2003) Ras-effector interactions: after one decade. Curr. Opin. Struct. Biol. 13, $122-129$

17 Bos, J. L., Rehmann, H. and Wittinghofer, A. (2007) GEFs and GAPs: critical elements in the control of small $G$ proteins. Cell $\mathbf{1 2 9}, 865-877$

18 Cherfils, J. and Zeghouf, M. (2013) Regulation of small GTPases by GEFs, GAPs, and GDIs. Physiol. Rev. 93, 269-309

19 Gillingham, A. K. and Munro, S. (2007) The small G proteins of the Arf family and their regulators. Annu. Rev. Cell Dev. Biol. 23, 579-611

20 Pasqualato, S., Renault, L. and Cherfils, J. (2002) Arf, Arl, Arp and Sar proteins: a family of GTP-binding proteins with a structural device for "front-back" communication. EMBO Rep. 3, 1035-1041

21 Pasqualato, S., Ménétrey, J., Franco, M. and Cherfils, J. (2001) The structural GDP/GTP cycle of human Arf6. EMBO Rep. 2, 234-238

22 Hori, Y., Kobayashi, T., Kikko, Y., Kontani, K. and Katada, T. (2008) Domain architecture of the atypical Arf-family GTPase Arl13b involved in cilia formation. Biochem. Biophys. Res. Commun. 373, 119-124

23 Humbert, M. C., Weihbrecht, K., Searby, C. C., Li, Y., Pope, R. M., Sheffield, V. C. and Seo, S. (2012) ARL13B, PDE6D, and CEP164 form a functional network for INPP5E ciliary targeting. Proc. Natl. Acad. Sci. U.S.A. 109, 19691-19696

24 Stolc, V., Samanta, M. P., Tongprasit, W. and Marshall, W. F. (2005) Genome-wide transcriptional analysis of flagellar regeneration in Chlamydomonas reinhardtii identifies orthologs of ciliary disease genes. Proc. Natl. Acad. Sci. U.S.A. 102, 3703-3707 
25 Veltel, S., Kravchenko, A., Ismail, S. and Wittinghofer, A. (2008) Specificity of Arl2/Arl3 signaling is mediated by a ternary Arl3-effector-GAP complex. FEBS Lett. $\mathbf{5 8 2}$, 2501-2507

26 Kühnel, K., Veltel, S., Schlichting, I. and Wittinghofer, A. (2006) Crystal structure of the human retinitis pigmentosa 2 protein and its interaction with Arl3. Structure 14, 367-378

27 Kabsch, W. (1993) Automatic processing of rotation diffraction data from crystals of initially unknown symmetry and cell constants. J. Appl. Chrystallogr. 26, 795-800

28 Collaborative Computational Project, Number 4 (1994) The CCP4 suite: programs for protein crystallography. Acta Crystallogr., Sect. D: Biol. Crystallogr. 50, 760-763

29 Hanzal-Bayer, M., Renault, L., Roversi, P., Wittinghofer, A. and Hillig, R. C. (2002) The complex of Arl2-GTP and PDE $\delta$ : from structure to function. EMBO J. 21, 2095-2106

30 Murshudov, G. N., Vagin, A. A. and Dodson, E. J. (1997) Refinement of macromolecular structures by the maximum-likelihood method. Acta Crystallogr., Sect. D: Biol. Crystallogr. 53, 240-255

31 Maurer, T. and Kalbitzer, H. R. (1996) Indirect referencing of ${ }^{31} \mathrm{P}$ and ${ }^{19} \mathrm{~F}$ NMR spectra. J. Magn. Reson. B 113, 177-178

32 Meierhofer, T., Eberhardt, M. and Spoerner, M. (2011) Conformational states of ADP ribosylation factor 1 complexed with different guanosine triphosphates as studied by ${ }^{31} \mathrm{P}$ NMR spectroscopy. Biochemistry 50, 6316-6327

33 Ménétrey, J., Macia, E., Pasqualato, S., Franco, M. and Cherfils, J. (2000) Structure of Arf6-GDP suggests a basis for guanine nucleotide exchange factors specificity. Nat. Struc. Mol. Biol. 7, 466-469

34 Gasper, R., Meyer, S., Gotthardt, K., Sirajuddin, M. and Wittinghofer, A. (2009) It takes two to tango: regulation of $G$ proteins by dimerization. Nat. Rev. Mol. Cell Biol. 10, 423-429

35 Spoerner, M., Herrmann, C., Vetter, I. R., Kalbitzer, H. R. and Wittinghofer, A. (2001) Dynamic properties of the Ras switch I region and its importance for binding to effectors. Proc. Natl. Acad. Sci. U.S.A. 98, 4944-4949

36 Seeburg, P. H., Colby, W. W., Capon, D. J., Goeddel, D. V. and Levinson, A. D. (1984) Biological properties of human c-Ha-ras1 genes mutated at codon 12. Nature $\mathbf{3 1 2}$ $71-75$

37 Ismail, S. A., Vetter, I. R., Sot, B. and Wittinghofer, A. (2010) The structure of an Arf-ArfGAP complex reveals a $\mathrm{Ca}^{2+}$ regulatory mechanism. Cell 141, 812-821

Received 16 August 2013/21 October 2013; accepted 30 October 2013

Published as BJ Immediate Publication 30 October 2013, doi:10.1042/BJ20131097
38 Hillig, R. C., Hanzal-Bayer, M., Linari, M., Becker, J., Wittinghofer, A. and Renault, L. (2000) Structural and biochemical properties show ARL3-GDP as a distinct GTP binding protein. Structure 8, 1239-1245

39 Veltel, S., Gasper, R., Eisenacher, E. and Wittinghofer, A. (2008) The retinitis pigmentosa 2 gene product is a GTPase-activating protein for Arf-like 3. Nat. Struc. Mol. Biol. 15, 373-380

40 Chiang, A. P., Nishimura, D., Searby, C., Elbedour, K., Carmi, R., Ferguson, A. L., Secrist, J., Braun, T., Casavant, T., Stone, E. M. et al. (2004) Comparative genomic analysis identifies an ADP-ribosylation factor - like gene as the cause of Bardet-Biedl syndrome (BBS3). Am. J. Hum. Genet. 75, 475-484

41 Fan, Y., Esmail, M. A., Ansley, S. J., Blacque, O. E., Boroevich, K., Ross, A. J., Moore, S. J., Badano, J. L., May-simera, H., Compton, D. S. et al. (2004) Mutations in a member of the Ras superfamily of small GTP-binding proteins causes Bardet-Biedl syndrome. Nat. Genet. 36, 989-993

42 Li, Y., Wei, Q., Zhang, Y., Ling, K. and Hu, J. (2010) The small GTPases ARL-13 and ARL-3 coordinate intraflagellar transport and ciliogenesis. J. Cell Biol. 189, 1039-1051

43 Ismail, S. A., Chen, Y.-X., Miertzschke, M., Vetter, I. R., Koerner, C. and Wittinghofer, A. (2012) Structural basis for Arl3-specific release of myristoylated ciliary cargo from UNC119. EMBO J. 31, 4085-4094

44 Zhang, T., Li, S., Zhang, Y., Zhong, C., Lai, Z. and Ding, J. (2009) Crystal structure of the ARL2-GTP-BART complex reveals a novel recognition and binding mode of small GTPase with effector. Structure 17, 602-610

45 Daumke, O., Weyand, M., Chakrabarti, P. P., Vetter, I. R. and Wittinghofer, A. (2004) The GTPase-activating protein Rap1GAP uses a catalytic asparagine. Nature 429, 197-201

46 Scrima, A., Thomas, C., Deaconescu, D. and Wittinghofer, A. (2008) The Rap-RapGAP complex: GTP hydrolysis without catalytic glutamine and arginine residues. EMBO J. 27, $1145-1153$

47 Pan, X., Eathiraj, S., Munson, M. and Lambright, D. G. (2006) TBC-domain GAPs for Rab GTPases accelerate GTP hydrolysis by a dual-finger mechanism. Nature 442, 303-306

48 Ménétrey, J., Perderiset, M., Cicolari, J., Dubois, T., Elkhatib, N., El Khadali, F., Franco, M., Chavrier, P. and Houdusse, A. (2007) Structural basis for ARF1-mediated recruitment of ARHGAP21 to Golgi membranes. EMBO J. 26, 1953-1962 


\section{SUPPLEMENTARY ONLINE DATA}

\section{Structural insights into the small G-protein Arl13B and implications for Joubert syndrome}

Mandy MIERTZSCHKE*, Carolin KOERNER*, Michael SPOERNER $\dagger$ and Alfred WITTINGHOFER ${ }^{\star 1}$

${ }^{*}$ Emeritus group A. Wittinghofer, Max-Planck-Institute for Molecular Physiology, BMZ, Otto-Hahn-Straße 15, 44227 Dortmund, Germany

$\dagger$ Institute of Biophysics and Physical Biochemistry, University of Regensburg, Universitätsstraße 31, 93053 Regensburg, Germany

Table S1 Data collection and refinement statistics of the CrArl13B$\mathrm{p}[\mathrm{NH}] p p \mathrm{G}$ structure determination (molecular replacement)

\begin{tabular}{|c|c|}
\hline Parameter & CrArl13B-p[NH]ppG \\
\hline \multicolumn{2}{|l|}{ Data collection } \\
\hline Space group & $P 2_{1} 2_{1} 2_{1}$ \\
\hline \multicolumn{2}{|l|}{ Cell dimensions } \\
\hline$a, b, c(\AA)$ & $48.05,76.83,172.92$ \\
\hline$\alpha, \beta, \gamma\left(^{\circ}\right)$ & $90.00,90.00,90.00$ \\
\hline Resolution $(\AA)$ & $19.82-2.50(2.6-2.5)$ \\
\hline$R_{\text {sym }}$ or $R_{\text {merge }}$ & $6.50(51.90)$ \\
\hline||$\sigma \mid$ & $30.03(5.78)$ \\
\hline Completeness (\%) & $99.7(100)$ \\
\hline Redundancy & 14.72 \\
\hline \multicolumn{2}{|l|}{ Refinement } \\
\hline Resolution (Å) & 2.5 \\
\hline Number of reflections & 21746 \\
\hline$R_{\text {work }} / R_{\text {tree }}$ & $0.1896 / 0.2353$ \\
\hline Number of atoms & 4921 \\
\hline Protein & 4808 \\
\hline Ligand/ion & 114 \\
\hline Water & 65 \\
\hline$B$-factors & 29.46 \\
\hline \multicolumn{2}{|l|}{ RMSD } \\
\hline Bond lengths $(\AA)$ & 0.014 \\
\hline Bond angles $\left({ }^{\circ}\right)$ & 1.758 \\
\hline
\end{tabular}

Table S2 Fitted values of ${ }^{31} \mathrm{P}$ NMR for Arl13B proteins

Chemical shift errors \pm 0.01 p.p.m. Error for line-width (total at half-height) $\pm 2 \mathrm{~Hz}$.

\begin{tabular}{|c|c|c|c|c|c|c|}
\hline Compound & \multicolumn{2}{|l|}{ Arl13B WT } & \multicolumn{2}{|c|}{ Arl113B R77Q } & \multicolumn{2}{|c|}{ Arl13B R194C } \\
\hline $\begin{array}{l}\text { GDP-Mg }{ }^{2+} \\
\text { GTP-Mg2+ }\end{array}$ & $\begin{array}{l}-9.35(\alpha) \\
-2.82(\beta) \\
-9.75(\alpha) \\
-16.10(\beta) \\
-6.58(\gamma)\end{array}$ & $\begin{array}{l}86(\alpha) \\
88(\beta) \\
\sim 86(\alpha) \\
64(\beta) \\
110(\gamma)\end{array}$ & $\begin{array}{l}-9.38(\alpha) \\
-2.83(\beta)\end{array}$ & $\begin{array}{l}120(\alpha) \\
84(\beta)\end{array}$ & $\begin{array}{l}-9.39(\alpha) \\
-2.81(\beta)\end{array}$ & $\begin{array}{l}80(\alpha) \\
64(\beta)\end{array}$ \\
\hline
\end{tabular}

1 To whom correspondence should be addressed (email alfred.wittinghofer@mpi-dortmund.mpg.de).

The structural co-ordinates reported for C-terminally truncated Arl13B from Chlamydomonas rheinhardtii bound to p[NH]ppG will appear in the PDB under code $4 \mathrm{M} 9 \mathrm{Q}$. 
Hs Arl13B Mm Ar113B Rn Arl13B Bt Arl13B Gg Ar113B Mamu Ar113: Xt Ā $113 B$ Dr Arl13B Ac Arl13B Am Arl13B Pt_Arl13 $\mathrm{Cj}^{-} \mathrm{Ar} 113 \mathrm{~B}$ Oa-Ar113B Cr_Ar113B

Hs Ar113B Mm-Ar113B Rn Arl13B Bt_Ar113B Gg_Arl13B Mamu_Arl13 Xt_Ar $113 B$ Dr-Ar113B AC Ar113B Am Ar113B Pt_Ar113B N1_Ar113B Cj_Ar113B Ca_Ar113B

Hs_Ar113B Mm Ar113B Rn Ar113B Bt Ar113B Gg_Ar113B Mamu_Ar113 Xt_Ā̄113B Dr_Ar113B Ac_Ar Ar113B Am Ar113B Pt_Ar113B N1_Ar113B Cj_Ar113B Oa Arl13B Cr_Ar113B

Hs Ar113B Mm_Ar113B Rn_Ar113B Bt_Ar113B Gg_Ar113B Mamu_Ar113 Xt_Ā $113 \mathrm{~B}$ Dr_Ar113B Ac_Ar113B Am_Ar113B Pt Ar113B N1_Ar113B $\mathrm{Cj}_{-}$Ar113B Oa_Ar113B Cr Ar113B

\section{0} * 20 * 20

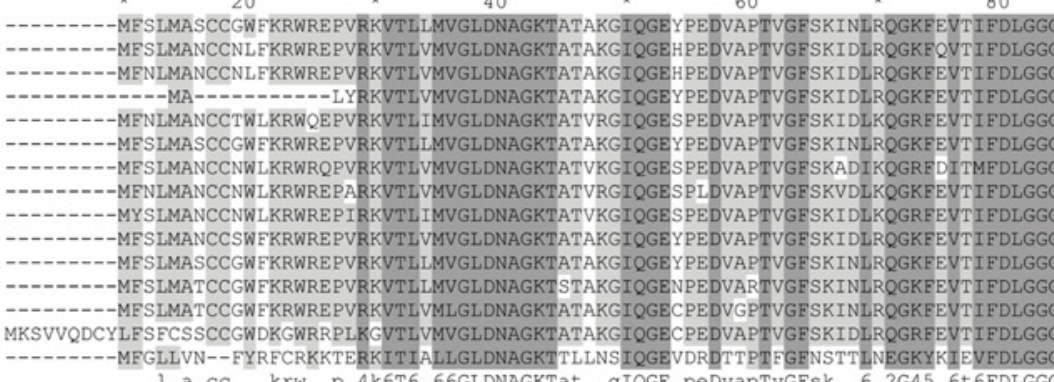

80

$\sqrt{3}$

100

IRIRGIWKNYYAESYGVIFVVDSSDËEERMEE : 106 KRIRGIWKNYYAESYGVIFVVDSSDEERMEE : 106 $:$
GRIRGIWKNYYAESYGVIFVVDSSDEDRMEE : GKRIRGIWKNYYAESYGVI FVVDSSDEERMEE : $\quad 91$ IRIRGIWKNYYAESYGVIFVVDSSDEERMEE : 106 GKRIRGIWKNYYAESYGVVFVIDSSDVERMEE : 106

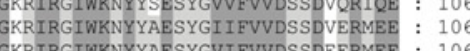
\begin{tabular}{ll} 
& \\
GKRIRGIWKNYYAESYGIIFVVDSSDVERMEE & $: 106$ \\
\hline GKRIRGIWKNYYAESYGVIFVVDSSDEERMEE & $: 106$
\end{tabular} $: 106$
GIRIRGIWKNYYAESYGVIFVVDSSDEERMEE GIRIRGIWKNYYAESYGVIFVVDSSDEERMEE
:

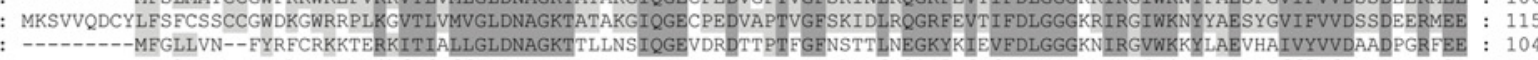

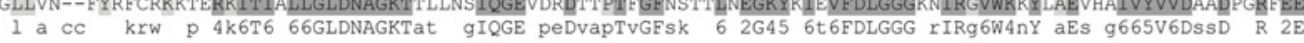

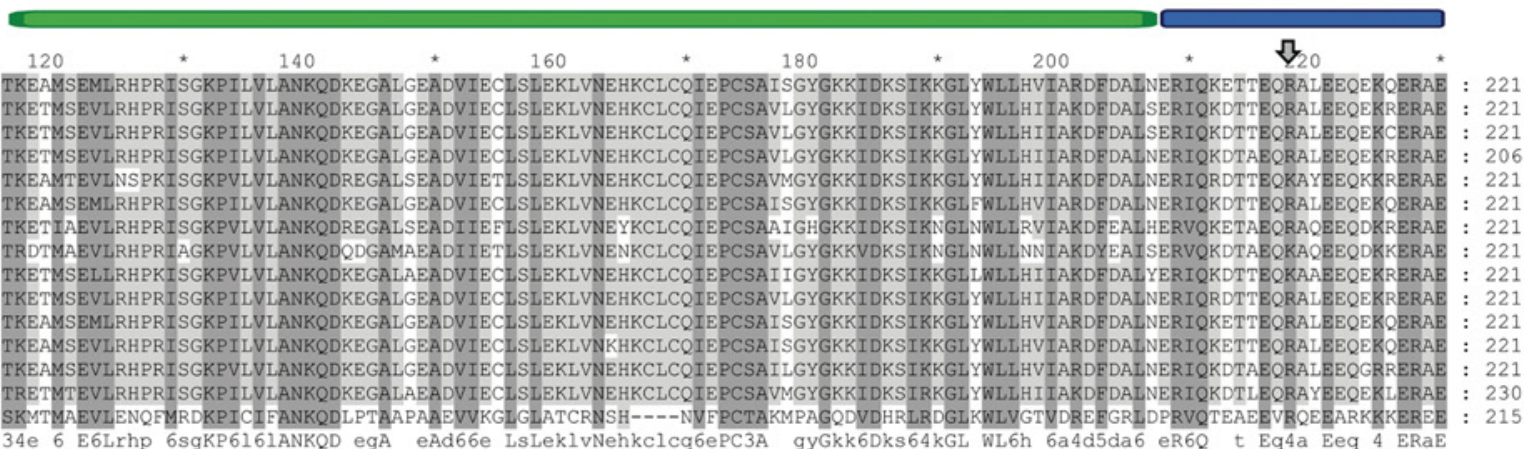

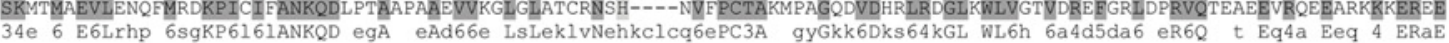

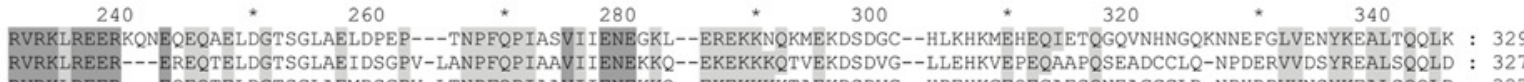
RVRKLREER---EQEQTELDGTSGLAEMDSGPV-LTNPFQPIAAVIIENEKKQ--EKEKKKKTAEKDSDVG--HPEHKGEQEGAESQNEAGGCLR-NPHRDVVNSYKEALSQQLD : 32 RVRKLREEREQREQEQAELHGTSGMAELDPEPV-IVNPFQPIASVIIENEKKL--EREKKRQNTE-DSDGC--PLKHKMEHEQIETQSQISDSSQKNSEFGVVENYKEALTQQLE : 31 S

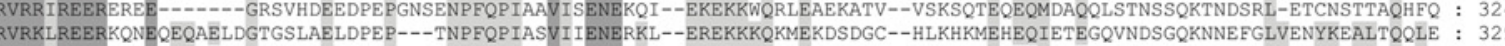
RVKRLREEREEKEKEEAGREGKQEIDEDTSIP--MVNPFQPISAVIIENEEKIRKEKEKKRQKMQTKQNGVGIALESKEEEDKMETGSESSQNSRTPRDNDLLESYKDALVQKLD : 334

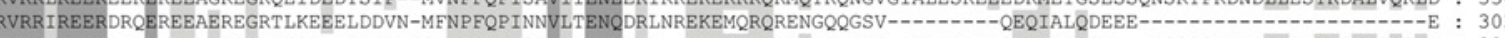
RVRKIREERERKEKEEAEREGRTISDDVDAELVIG-NPFQPISTVIAENEKKI--EKEKKRQREENEKEVL--VLKPKTNQEQMDTQSL---ISQQSNDYRLVEKYKCALSQQLDD : 32 RVRKLREEREQREREQAEMNGTSSLAELDPEPI-IVNPFQPIASVILENERKL--EKEKTRQNMEKDSDGG--PLKHKVEHEQIETQSQISDSSQKNNELGIVENYKEALTQQLE : 33

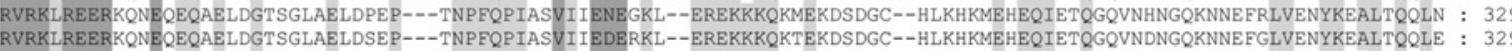
RVRKLREERKQNQQEQAELDGTSGRAELDPEP---MNPFQPIASVII IENERKL--EKEKKKQKMETDSDGC--PLKPKMEPEQMETQGQVNDSGQKNNEFGLLENYKESLTQQLE : 329 RVKKLREEREQREREEAEHEGRNRPFEVETEPI-VNNPFQPIAAVILENEKKT--EKEKKKQMMESGRDDT--PLKSNPEQEQMETQSHTSCSSQKINECGLVETYKMALTQQLE : 340 R644 REER 2 e g e p npfqpi V E12 e ekk q e eq al ${ }^{2}$
\end{abstract}

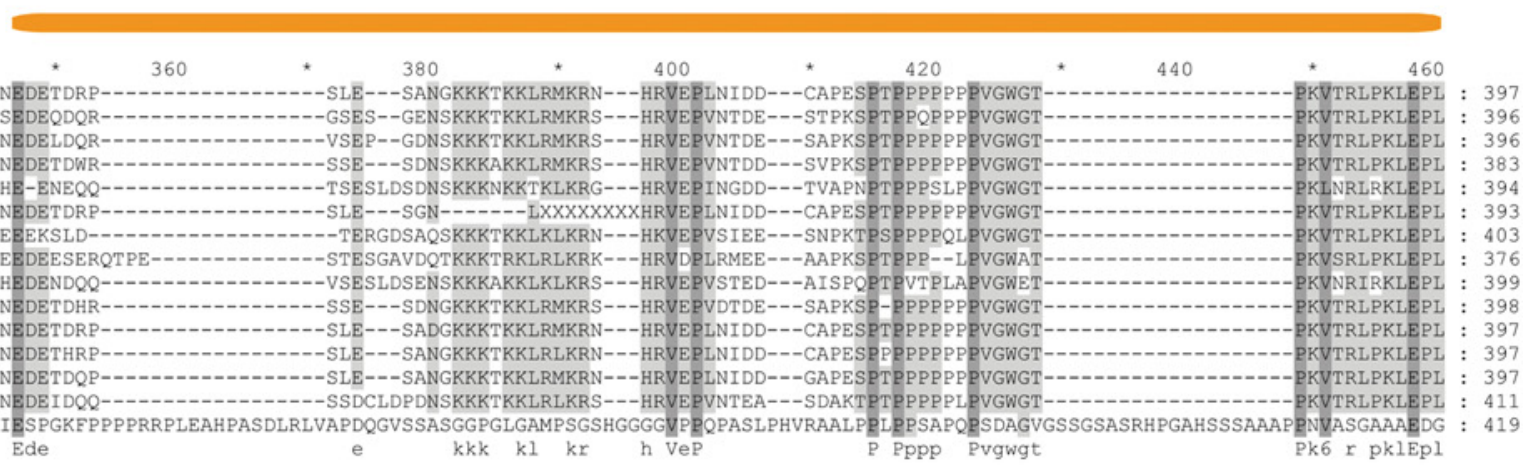

$\begin{array}{llccc}* & & 480 & *\end{array}$



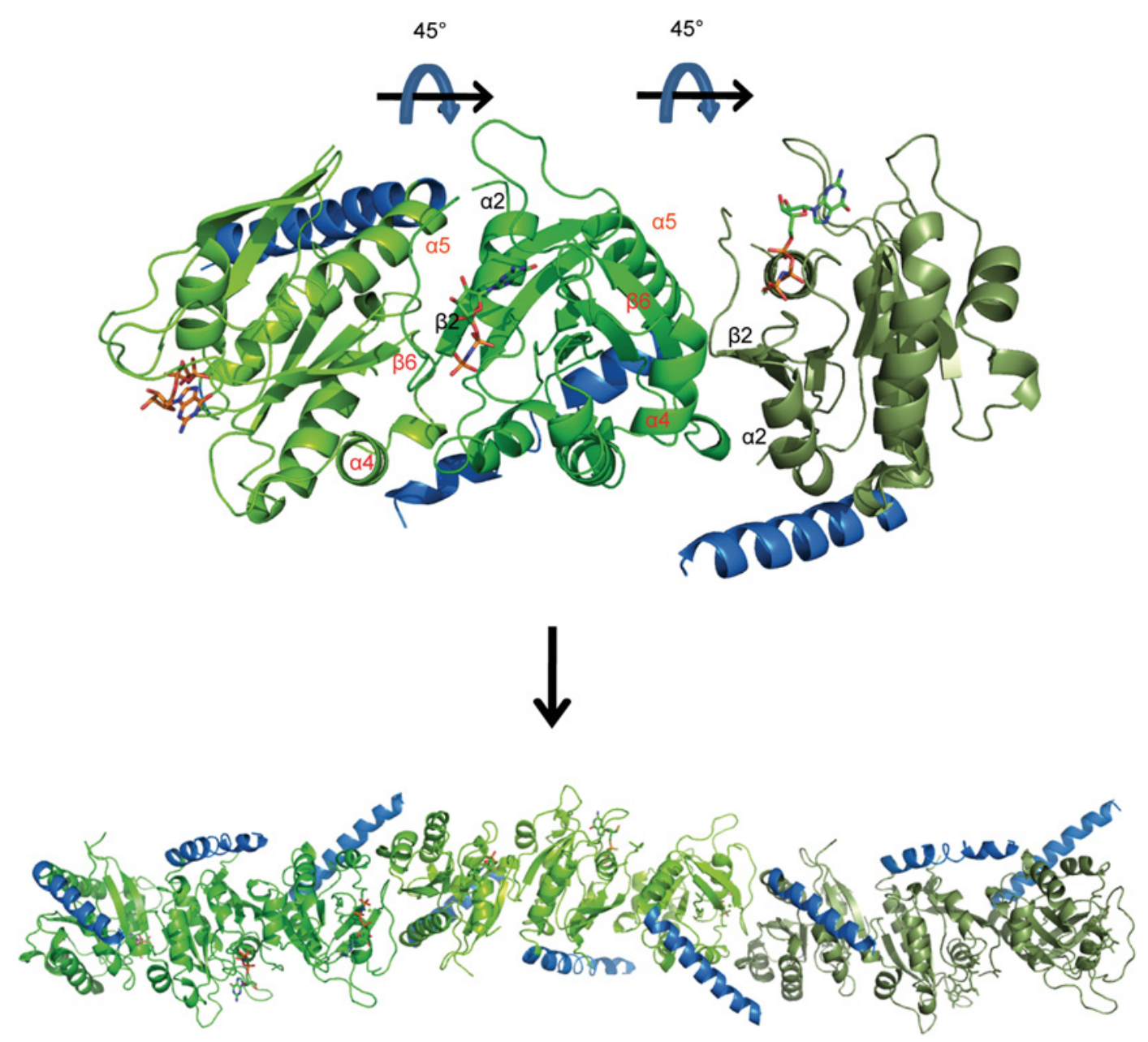

Figure S2 Arrangement of CrArl13B crystal in $P 2_{1} 2_{1} 2_{1}$

Three CrArl13B monomers are shown in the asymmetric unit (upper panel). The G-domain is coloured in green and the CC region in blue. The monomers are rotated by $45^{\circ}$ in respect to each other. The secondary structure elements involved in contacts between the monomers are indicated. Within the crystal, a fibre-like arrangement (lower panel) can be observed.

\section{Figure S1 Alignment of Arl13B proteins}

Arl13B proteins are shown from Homo sapiens (Hs), Mus musculus (Mm), Rattus norvegicus (Rn), Bos taurus (Bt), Gallus gallus (Gg), Macaca mulatta (Mamu), Xenopus tropicales (Xt), Danio rerio (Dr), Anolis carolinensis (Aa), Ailuropoda melanoleuca (Am), Pan troglodytes (Pt), Nomascus leucogenys (NI), Callithrix jacchus (Cj), Ornitharhyndus anaticus (Oa) and C. rheinhartii (Cr). Marked above are the G-domain in green, the CC region in blue and the PRR in orange. 

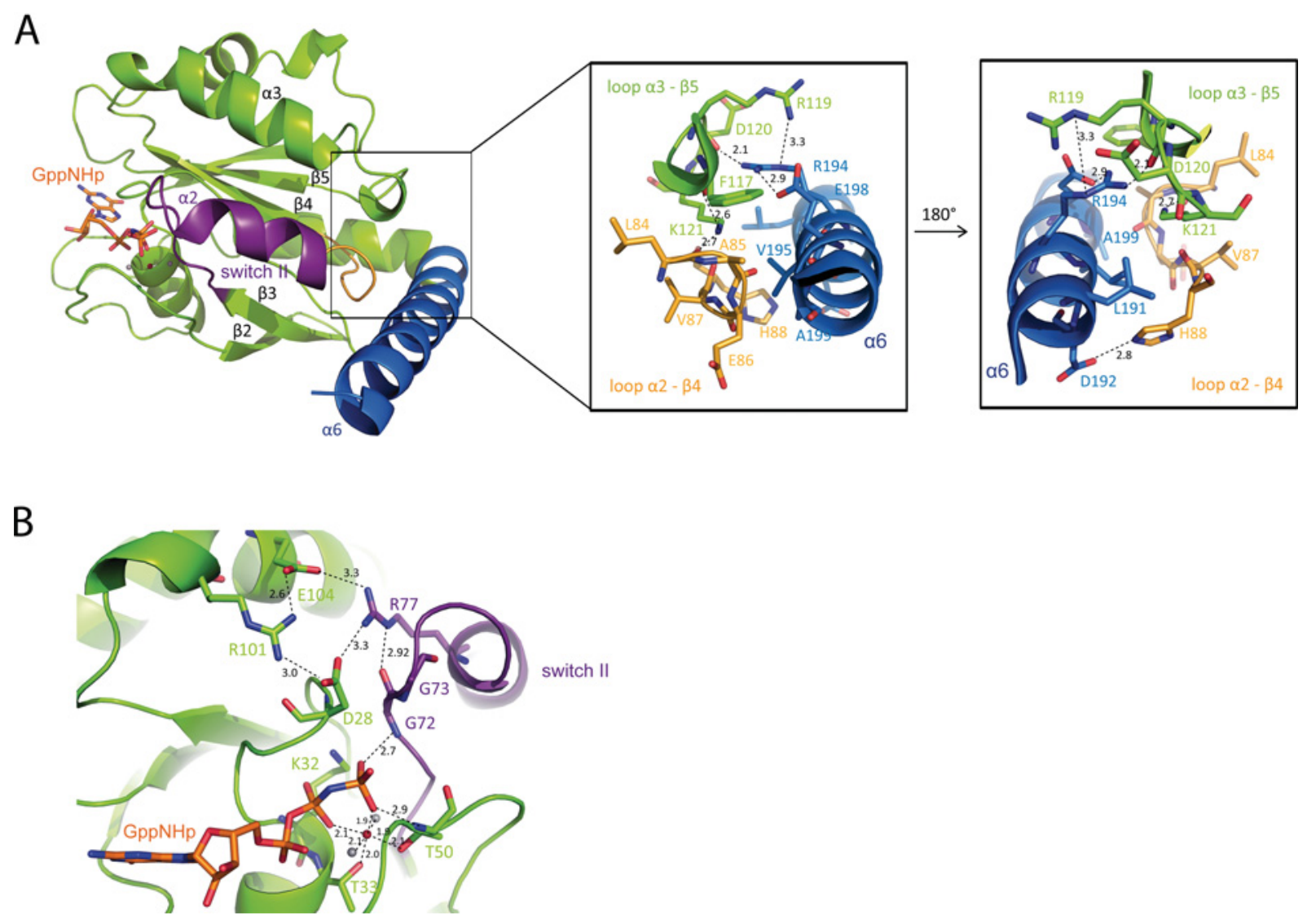

\section{Figure S3 Interaction details}

(A) Interface of the G-domain and helix $\alpha 6$ of CrArl13B. The main interaction of the G-domain with $\alpha 6$ is mediated by a loop connecting $\alpha 2$ and $\beta 4$ and a loop linking $\alpha 3$ and $\beta 5$ of the G-domain. The main residues and distances (in $\AA$ ) are shown. The zoom (right-hand panels) into the interaction inferface is shown by two views rotated $180^{\circ}$. (B) Next to the interactions of the $\gamma$-phosphate with residues of the G-domain, the interactions of $\mathrm{Arg}^{77}$ of switch $I 1$ are shown. The main residues and distances (in $\AA$ ), water ions in grey and $\mathrm{Mg}^{2}+$ in red are shown.

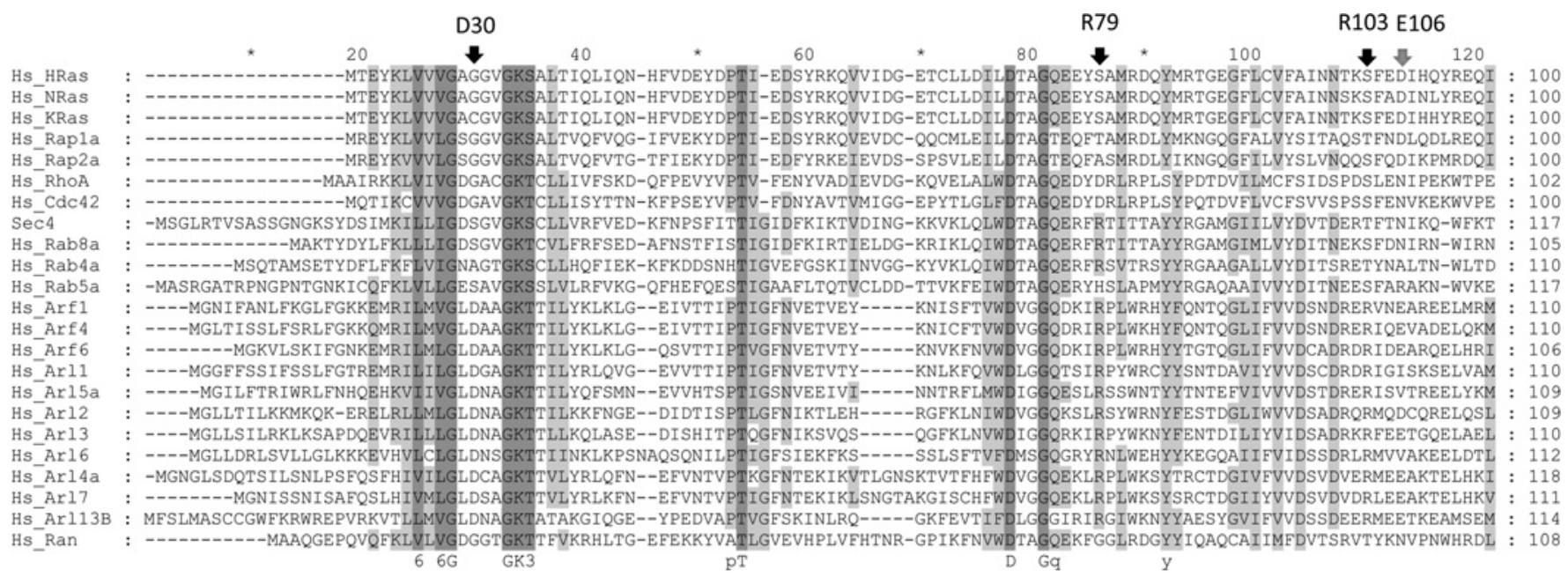

\section{Figure S4 Alignment of several human (Hs) small G-proteins including few examples of each subfamily}

Conserved residues are highlighted in dark and light grey depending on their degree of conservation. Highlighted in cyan and marked by a red arrow are the aspartate (P-loop), arginine ( $\alpha 5$ helix) and arginine ( $\alpha 2$ helix) residues involved in the interaction network shown in Figure 7(A) of the main text. 

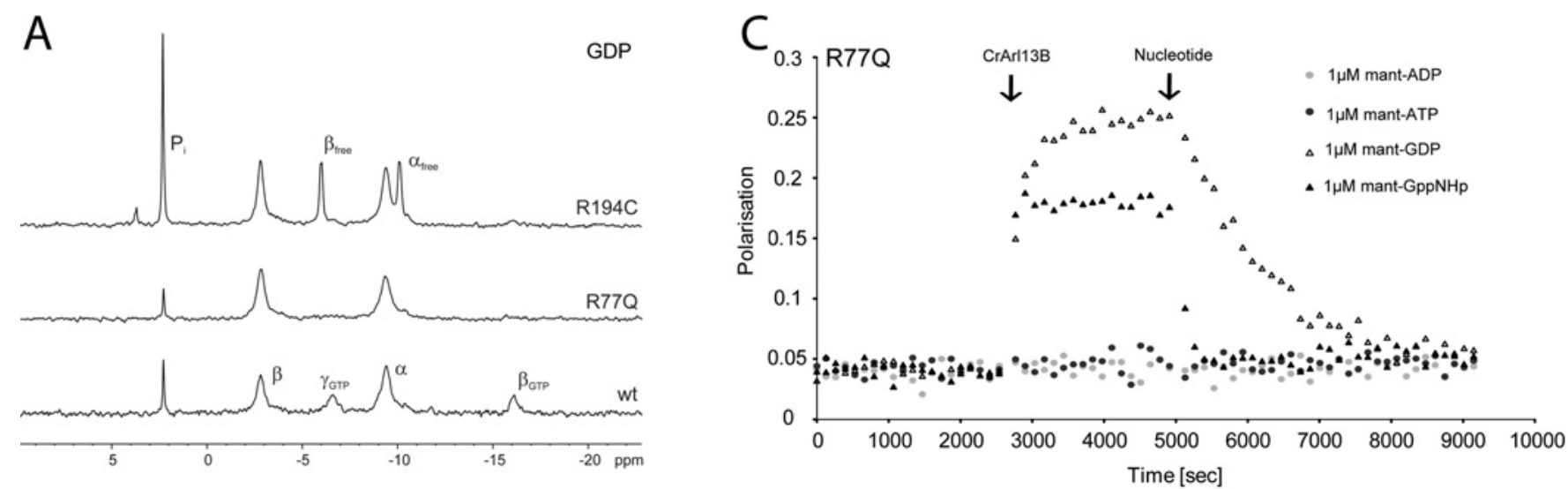

B

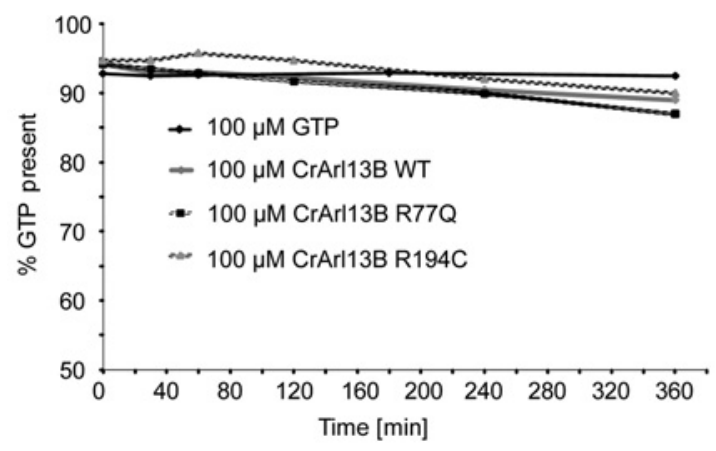

$\mathrm{D}$

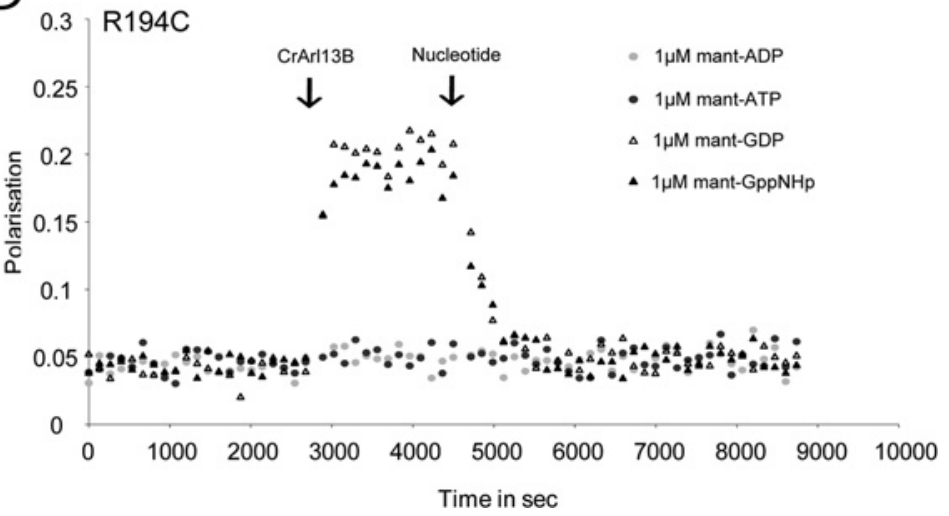

Figure S5 Patient mutations R77Q and R194C

(A) ${ }^{31}$ P NMR spectra of 1-1.5 mM Mg${ }^{2+}$.GDP complexes of CrArl13B ${ }^{\text {WT }}$, CrArl13B ${ }^{\text {R770 }}$ and CrArl13B B194C at $278 \mathrm{~K}$ in buffer M. Signals are assigned according to Meierhofer et al. [1]. (B) Hydrolysis of GTP bound to CrAr13B wild-type, R77Q and R194C is monitored over time. (C and D) Fluorescence polarization measurement at $20^{\circ} \mathrm{C}$ in buffer M. To $1 \mu \mathrm{M}$ mant-labelled nucleotides $\{$ ADP, ATP, GDP and p[NH]ppG (GppNHp) as indicated $\}$ were added $10 \mu \mathrm{M}$ CrArl13B R77Q (C) or R194C (D) respectively (first black arrow) and $400 \mu$ M unlabelled nucleotide (second black arrow).
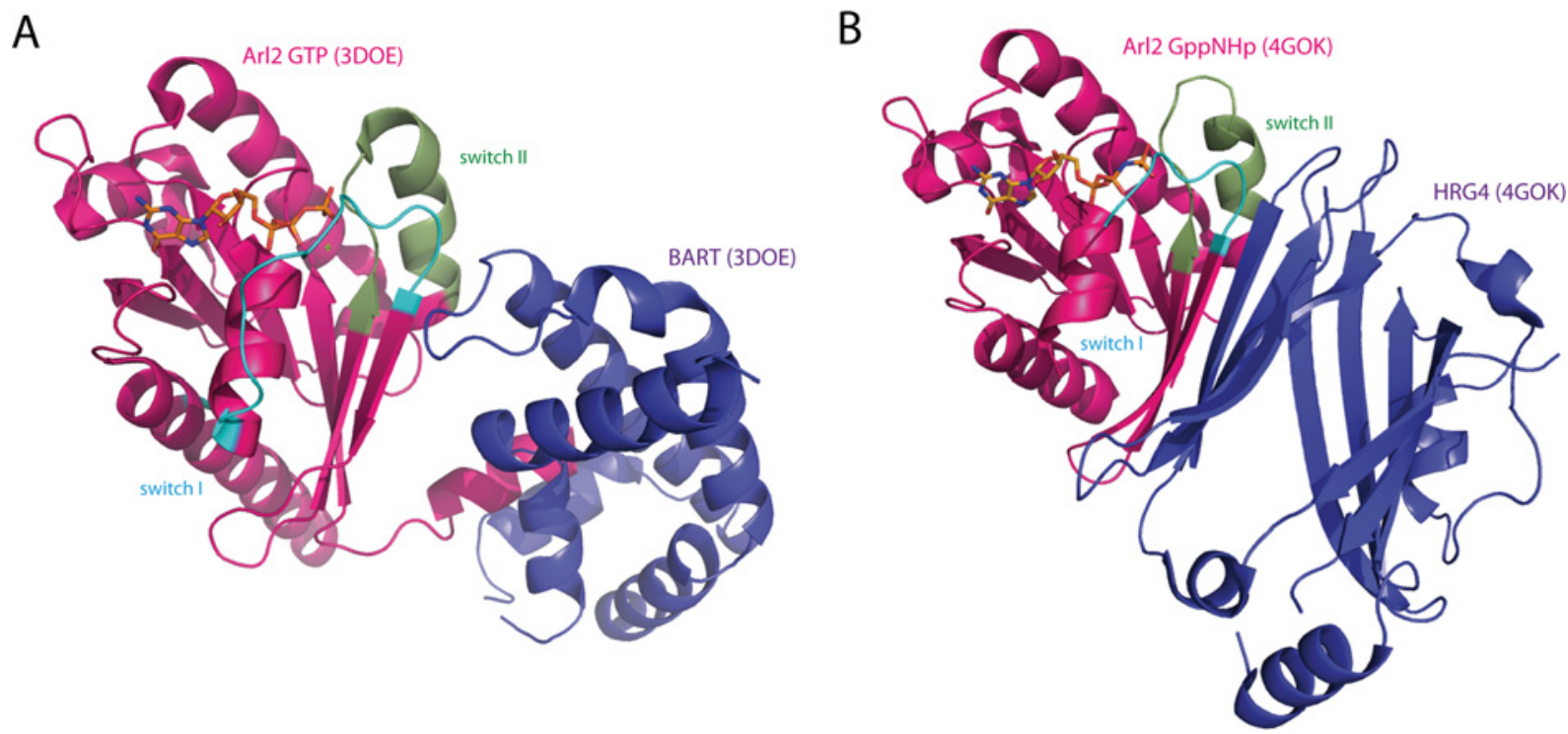

Figure S6 Arl effector interactions

(A) Crystal structure of Arl2-GTP-BART (PDB code 3DOE [2]). BART binds to the N-terminal helix of Arl2-GTP and contacts switch I (light blue) whereas minor contacts are made to switch II. (B) Crystal structure of Arl2-GppNHp-HRG4 (PDB code 4GOK [3]) shows that HRG4 is interacting with switch I and II. 


\section{REFERENCES}

1 Meierhofer, T., Eberhardt, M. and Spoerner, M. (2011) Conformational states of ADP ribosylation factor 1 complexed with different guanosine triphosphates as studied by ${ }^{31} \mathrm{P}$ NMR spectroscopy. Biochemistry $\mathbf{5 0}, 6316-6327$

2 Zhang, T., Li, S., Zhang, Y., Zhong, C., Lai, Z. and Ding, J. (2009) Crystal structure of the ARL2-GTP-BART complex reveals a novel recognition and binding mode of small GTPase with effector. Structure 17, 602-610

3 Ismail, S. A., Chen, Y.-X., Miertzschke, M., Vetter, I. R., Koerner, C. and Wittinghofer, A. (2012) Structural basis for Arl3-specific release of myristoylated ciliary cargo from UNC119. EMBO J. 31, 4085-4094

Received 16 August 2013/21 October 2013; accepted 30 October 2013

Published as BJ Immediate Publication 30 October 2013, doi:10.1042/BJ20131097 